\title{
Ornithofauna and its conservation in the Kuttanad wetlands, southern portion of Vembanad-Kole Ramsar site, India
}

\author{
S. Prasanth Narayanan ${ }^{1}$, A.P. Thomas ${ }^{2} \&$ B. Sreekumar ${ }^{3}$ \\ ${ }^{1,2}$ Advanced Centre of Environmental Studies and Sustainable Development (ACESSD), School of Environmental Sciences, \\ Mahatma Gandhi University, Priyadarsini Hills, Kottayam, Kerala 686560, India \\ ${ }^{3}$ Kottayam Nature Society, Sri Nilayam, Near Union Club, Kottayam, Kerala 686001, India \\ Email: ${ }^{1}$ narayanankc@gmail.com (corresponding author)
}

Date of publication (online): 26 April 2011 Date of publication (print): 26 April 2011 ISSN 0974-7907 (online) | 0974-7893 (print)

Editor: P.O. Nameer

\section{Manuscript details:}

Ms \# 01870

Received 10 October 2007

Final received 14 March 2011

Finally accepted 18 March 2011

Citation: Narayanan, S.P., A.P. Thomas \& B. Sreekumar (2011). Ornithofauna and its conservation in the Kuttanad wetlands, southern portion of Vembanad-Kole Ramsar site, India. Journal of Threatened Taxa 3(4): 1663-1676.

Copyright: (C) S. Prasanth Narayanan, A.P. Thomas \& B. Sreekumar 2011. Creative Commons Attribution 3.0 Unported License. JoTT allows unrestricted use of this article in any medium for non-profit purposes, reproduction and distribution by providing adequate credit to the authors and the source of publication.

For Author Details and Author Contribution see end of this article.

Acknowledgements: We are indebted to $\mathrm{Dr}$ V.P. Sylas and to the members of Kottayam Nature Society, who shared information and accompanying us during the field visits. We are also grateful to C. Sashikumar, J. Praveen and J. Ranjini for valuable comments and correction on the earlier drafts of the manuscript. We also thank the anonymous referees for comments that improved the manuscript.
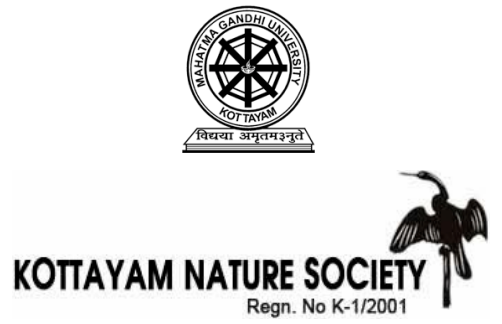

(c) (7) (口)

OPEN ACGESS | FREE DOWNLOAD
Abstract: The avifauna of Kuttanad was studied from January 1995 to June 2007. Two-hundred-and-twenty-five taxa of birds belonging to 15 orders and 59 families were recorded. Among the birds recorded, 38\% were migrants. Fifty-five species were found to breed in the area. Family Scolopaceidae showed maximum species diversity. European Roller Coracias garrulus recorded during this study is the first report of this species from Kerala. Ten globally threatened species were recorded. Kuttanad wetland shows greater species diversity, especially in the wetland birds, than the Kole wetlands of Kerala. Kumarakom heronry holds $8 \%$ of the biogeographical population of the Near Threatened Oriental Darter. Landscape alteration, hunting, felling of nesting trees and pesticides are the major detrimental factors for the survival of birds. Conservation aspects of birds of this region are discussed.

Keywords: Avifauna, conservation, Kuttanad, Ramsar site, threats

\section{INTRODUCTION}

Wetlands are complex and productive ecosystems (Maltby 1986; Unni 2002) that occupy about six percent of the Earth's land surface (Maltby \& Turner 1983). Wetlands are known as "biological supermarkets" because of the extensive food chains and rich biodiversity they support, providing unique habitats for a wide range of flora and fauna (Mitsch \& Gosselink 2000). Wetlands are important habitats for birds, which use them for feeding, roosting, nesting and rearing young (Weller 1999; Stewart 2001). The use of wetlands by birds during the breeding cycle ranges widely, with some depending almost totally on wetlands for breeding, feeding or shelter during their breeding cycles.

Kuttanad wetland is located at the southern portion of India's largest Ramsar site the Vembanad-Kole wetland. Ali (1984), Ali \& Ripley (1987), Neelakantan (1996), Chandy (2003), Narayanan (2004), Sreekumar \& Narayanan (2004), Rakesh et al. (2004), Narayanan et al. (2005a,b) reported various aspects of the avifauna of this wetland. The only detailed study regarding birds in this region is the midwinter water bird count. Nature Education Society, Thrissur, organized the first water bird survey in the Vembanad Lake (NEST 1993). Since 2001, regular Midwinter Waterbird Count is being carried out at different parts of Kuttanad wetlands by Kottayam Nature Society (KNS) in association with Kerala Forest and Wildlife Department (Sreekumar 2001, 2002, 2003, 2004, 2005), but most of the surveys were concentrated in and around the premises of Vembanad Lake. Existing information available on the avifauna of 
this region is based on surveys conducted by KNS and mainly in view to the wetland birds. Hence this work was taken with the following objectives (i) to make an inventory of the avifauna of Kuttanad wetlands with breeding birds, status, occurrence, (ii) to find and list the major factors which threatens the bird fauna, and (iii) to propose the action plan for the conservation of birds and wetlands of Kuttanad.

\section{STUDY AREA}

Kuttanad is primarily a deltaic formation of five river systems: Meenachil, Pamba, Manimala, Muvattupuzha and Achencovil, located in the fertile low-lying areas of Vembanad Lake (Fig. 1) (Shari \& Chitra 2005). It spreads over Alappuzha, Kottayam and Pathanamthitta districts of Kerala and forms an integral part of the Vembanad-Kole Ramsar site. This region lies between $9^{0} 17^{\prime}-9^{0} 40^{\prime} \mathrm{N} \& 76^{0} 19^{\prime}-76^{0} 33^{\prime} \mathrm{E}$ and is separated from

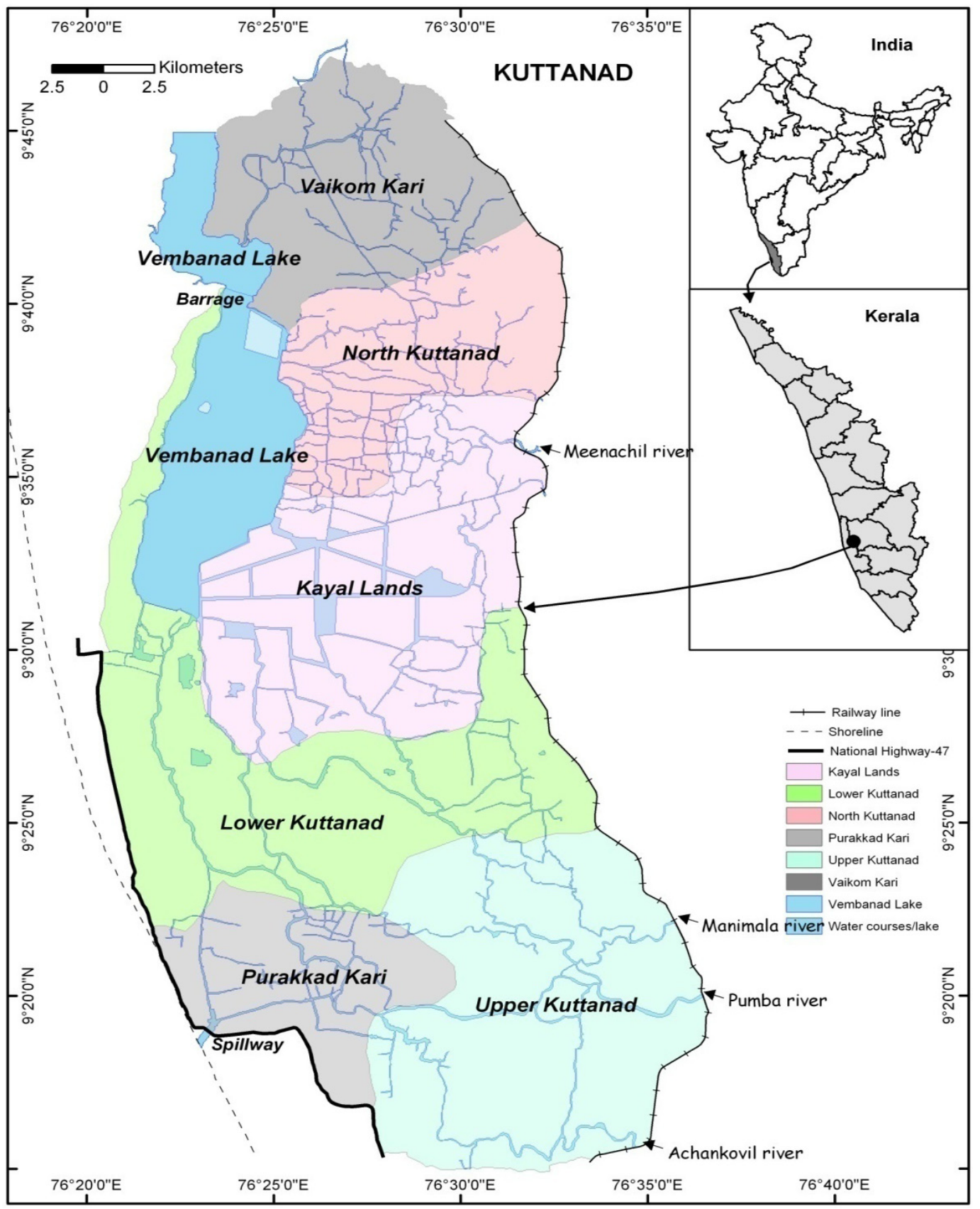

Figure 1. Six divisions of Kuttanad wetlands 
the Arabian Sea by a narrow strip of land. Much of this region lies 0.6 to $2.2 \mathrm{~m}$ below mean sea level, hence the area remains water-logged almost throughout the year and is subjected to continued flood submergence during the monsoon and saline water ingression during the summer months. Kuttanad is rightly called the "Rice Bowl of Kerala", contributing nearly 20\% of the total state rice production. It consists of 53,639 hectares distributed among 1086 units where rice is cultivated (Sudhikumar \& Sebastian 2005). Most of these fields are inundated during the non-crop season and water has to be pumped out to the canal systems and backwaters before the commencement of the cultivating season (Sashikumar \& Palot 2002). Based on the soils, geomorphology and salinity intrusion, Kuttanad is subdivided into six agro-ecological zones viz., (i) Upper Kuttanad (ii) Kayal lands (iii) Vaikom Kari (iv) Lower Kuttanad (v) North Kuttanad and (vi) Purakkad Kari (Indo-Dutch Mission 1989). Major portion of the Vembanad estuary is situated in Kuttanad Wetland, which is the biggest estuary in the southwest coast of India. It experiences warm climate with fairly uniform temperature throughout the year ranging from $21-36{ }^{\circ} \mathrm{C}$. Humidity in general is very high all through the year (Shari \& Chitra 2005). The average annual rainfall received is around $3000 \mathrm{~mm}$ (Shari \& Chitra 2005 ) of which about $83 \%$ is received during south west monsoon months.

\section{MATERIAL AND METHODS}

This study was carried out from January 1995 to June 2007. Observations were carried out during the weekdays mainly from 0700 to $1100 \mathrm{hr}$ and occasional sightings of birds during non-birding trips were also included. Birds were identified with the help of different field guides (Ali 1984; Ali \& Ripley 1987; Neelakantan 1996; Grimmet et al. 2000; Grewal et al. 2002) using Bushnell $(7 \times 35 \mathrm{~mm})$ binoculars. The species list (Table 1) includes those that were recorded in the present study and also from various other works, compiled from published and unpublished materials and personal communications. As per the occurrence in the Kuttanad wetlands, species were classified as: resident $(\mathrm{R})$ - found in all suitable habitats throughout the year; migrant (M) - found only during a specific season (this includes birds from Central
Asian countries and northern areas of the Indian subcontinent); local migrant (LM) - resident to the state but found in Kuttanad region only during a specific season; straggler from the hill ( $\mathrm{SH}$ ) - species usually found in the hilly areas of the state but recorded from this area; and vagrant (V) - birds which accidentally came to the region from its normal range, which is hundreds of miles away. The status of many birds oberved in Kuttanad Wetland is different from their Kerala State status. Abundance of each species was derived following Nameer et al. (2000). According to the feeding habits, birds were divided as aquatic herbivores, aquatic insectivore, aquatic omnivores, piscivores, carnivores, insectivores, omnivores, granivores, frugivores, nectarivores. The taxonomical classification and common names follow Manakadan $\&$ Pittie (2002).

\section{RESULTS AND DISCUSSION}

Total 225 taxa of birds belonged to 15 orders 59 families were identified from Kuttanad wetlands (Table 1). Neelakantan (1996) listed out 483 species from Kerala out of which around $47 \%$ of bird species were recorded during this study. Order Passeriformes posses the most diversified families (26) and species (78). Maximum number of species was recorded from the families Scolopaceidae and Ardeidae (Table 1). Though a wetland-dominated area, $52.5 \%$ birds belong to non-wetland category. Most of the land birds were seen at the eastern boundaries of Kuttanad, where Kuttanad wetlands meet midland areas of Kerala. Hence high number of species reported from that area could be due to edge effect. Among the birds recorded, $38 \%$ constitute migrants and 38\% residents (Fig. 2). Wetland and wetland dependent birds formed major portion of these migrants. The composition of birds in major feeding guilds in the study area showed that the insectivore guild was the most common with $37.33 \%$ species, followed by piscivores (Fig. 3).

Ten globally threatened species were recorded. Among these Greater Spotted Eagle Aquila clanga is listed under Vulnerable category. Species, such as Ferruginous Pochard Aythya nyroca, Painted Stork Mycteria leucocephala, Oriental White Ibis Threskiornis melanocephalus, Spot-billed Pelican Pelecanus philippensis, Oriental Darter Anhinga 
Table 1. Checklist of the birds of Kuttanad wetlands with its status

\begin{tabular}{|c|c|c|c|c|c|c|c|c|c|}
\hline & Scientific name & Common name & Status & $\begin{array}{l}\text { Bree- } \\
\text { ding }\end{array}$ & & Scientific name & Common name & Status & $\begin{array}{l}\text { Bree- } \\
\text { ding }\end{array}$ \\
\hline & Podicipedidae & & & & 25 & $\begin{array}{l}\text { Platalea leucorodia } \\
\text { Linnaeus, } 1758\end{array}$ & $\begin{array}{l}\text { Eurasian } \\
\text { Spoonbill }\end{array}$ & $\mathrm{U}, \mathrm{M}$ & \\
\hline 1 & $\begin{array}{l}\text { Tachybaptus ruficollis } \\
\text { (Pallas, 1764) }\end{array}$ & Little Grebe & $\mathrm{C}, \mathrm{R}$ & $\sqrt{ }$ & & Anatidae & & & \\
\hline & Pelecanidae & & & & 26 & $\begin{array}{l}\text { Dendrocygna javanica } \\
\text { (Horsfield, 1821) }\end{array}$ & $\begin{array}{l}\text { Lesser Whistling- } \\
\text { Duck }\end{array}$ & $C, R$ & $\sqrt{ }$ \\
\hline \multirow[t]{2}{*}{2} & $\begin{array}{l}\text { Pelecanus philippensis } \\
\text { Gmelin, } 1789\end{array}$ & $\begin{array}{l}\text { Spot-billed } \\
\text { Pelican }\end{array}$ & $\mathrm{O}, \mathrm{M}$ & & 27 & \multirow{2}{*}{$\begin{array}{l}\text { Tadorna ferruginea } \\
\text { (Pallas, 1764) }^{\#}\end{array}$} & \multirow{2}{*}{$\begin{array}{l}\text { Brahminy } \\
\text { Shelduck \# }\end{array}$} & v & \\
\hline & Phalacrocoracidae & & & & & & & & \\
\hline 3 & $\begin{array}{l}\text { Phalacrocorax niger } \\
\text { (Vieillot, 1817) }\end{array}$ & Little Cormorant & $A, R$ & $\sqrt{ }$ & 28 & $\begin{array}{l}\text { coromandelianus (Gmelin, } \\
1789 \text { ) }\end{array}$ & Cotton Teal & $C, R$ & $\sqrt{ }$ \\
\hline 4 & $\begin{array}{l}\text { Phalacrocorax fuscicollis } \\
\text { Stephens, } 1826\end{array}$ & Indian Shag & $C, R$ & $\sqrt{ }$ & 29 & $\begin{array}{l}\text { Anas poecilorhyncha J.R. } \\
\text { Forester, } 1781\end{array}$ & Spot-billed Duck & U, LM? & \\
\hline \multirow[t]{2}{*}{5} & $\begin{array}{l}\text { Phalacrocorax carbo } \\
\text { (Linnaeus, 1758) }\end{array}$ & Great Cormorant & U, R & $\checkmark$ & 30 & $\begin{array}{l}\text { Anas clypeata Linnaeus, } \\
1758\end{array}$ & $\begin{array}{l}\text { Northern } \\
\text { Shoveller }\end{array}$ & $\mathrm{O}, \mathrm{M}$ & \\
\hline & Anhingidae & & & & 31 & $\begin{array}{l}\text { Anas acuta Linnaeus, } \\
1758\end{array}$ & Northern Pintail & $\mathrm{C}, \mathrm{M}$ & \\
\hline \multirow[t]{2}{*}{6} & $\begin{array}{l}\text { Anhinga melanogaster } \\
\text { Pennant, } 1769\end{array}$ & Darter & $\mathrm{C}, \mathrm{R}$ & $\checkmark$ & 32 & $\begin{array}{l}\text { Anas querquedula } \\
\text { Linnaeus, } 1758\end{array}$ & Garganey & $\mathrm{C}, \mathrm{M}$ & \\
\hline & Ardeidae & & & & \multirow[b]{2}{*}{33} & \multirow{2}{*}{$\begin{array}{l}\text { Anas crecca Linnaeus, } \\
1758\end{array}$} & \multirow{2}{*}{ Common Teal } & \multirow{2}{*}{ U, M } & \\
\hline 7 & $\begin{array}{l}\text { Egretta garzetta } \\
\text { (Linnaeus, 1766) }\end{array}$ & Little Egret & $A, R$ & $\sqrt{ }$ & & & & & \\
\hline 8 & $\begin{array}{l}\text { Egretta gularis (Bosc, } \\
1792 \text { ) }\end{array}$ & $\begin{array}{l}\text { Western Reef- } \\
\text { Egret }\end{array}$ & $\mathrm{U}, \mathrm{M}$ & & 34 & $\begin{array}{l}\text { Aythya nyroca } \\
\text { (Guldenstadt, 1770) }\end{array}$ & $\begin{array}{l}\text { Ferruginous } \\
\text { Pochard }\end{array}$ & $\mathrm{O}, \mathrm{M}$ & \\
\hline & Ardea cinerea Linnaeus, & & & & & Accipitridae & & & \\
\hline 9 & 1758 & Grey Heron & U, LM & & 35 & \begin{tabular}{|l} 
Elanus caeruleus \\
(Desfontaines, 1789)
\end{tabular} & $\begin{array}{l}\text { Black-shouldered } \\
\text { Kite }\end{array}$ & U, LM & \\
\hline 10 & $\begin{array}{l}\text { Ardea purpurea Linnaeus, } \\
1766\end{array}$ & Purple Heron & $C, R$ & $\sqrt{ }$ & 36 & \begin{tabular}{|l|} 
Milvus migrans (Boddaert, \\
1783 )
\end{tabular} & Black Kite & $U, \mathrm{R}$ & $\sqrt{ }$ \\
\hline 11 & $\begin{array}{l}\begin{array}{l}\text { Casmerodius albus } \\
\text { (Linnaeus, 1758) }\end{array} \\
\end{array}$ & Large Egret & $C, R$ & $\checkmark$ & 37 & $\begin{array}{l}\text { Haliastur indus (Boddaert, } \\
\text { 1783) }\end{array}$ & Brahminy Kite & $\mathrm{C}, \mathrm{R}$ & $\sqrt{ }$ \\
\hline 12 & $\begin{array}{l}\text { Mesophoyx intermedia } \\
\text { (Wagler, 1829) }\end{array}$ & Median Egret & $C, R$ & $\sqrt{ }$ & 38 & $\begin{array}{l}\text { Circus } \\
\text { aeruginosus(Linnaeus }\end{array}$ & Western Marsh- & C. M & \\
\hline 13 & Bubulcus ibis (Linnaeus, & Cattle Egret & $A, M$ & & & 1758) & Harrier & & \\
\hline 14 & Ardeola grayii (Sykes, & & $A P$ & $\sqrt{1}$ & 39 & $\begin{array}{l}\text { Circus pygargus } \\
\text { (Linnaeus, 1758) } \#\end{array}$ & $\begin{array}{l}\text { Montagu's } \\
\text { Harrier \# }\end{array}$ & $\mathrm{O}, \mathrm{M}$ & \\
\hline 14 & 1832) & Heron & $\mathrm{A}, \mathrm{N}$ & $v$ & & & Greater Grey- & & \\
\hline 15 & $\begin{array}{l}\text { Butorides striatus } \\
\text { (Linnaeus, 1758) }\end{array}$ & $\begin{array}{l}\text { Little Green } \\
\text { Heron }\end{array}$ & U, R & & 40 & (Horsfield, 1821) $\#$ & $\begin{array}{l}\text { headed Fish- } \\
\text { Eagle\# }\end{array}$ & $\mathrm{O}, \mathrm{SH}$ & \\
\hline 16 & $\begin{array}{l}\text { Nycticorax nycticorax } \\
\text { (Linnaeus, 1758) }\end{array}$ & $\begin{array}{l}\text { Black-crowned } \\
\text { Night-Heron }\end{array}$ & $C, R$ & $\checkmark$ & 41 & $\begin{array}{l}\text { Accipter badius (Gmelin, } \\
\text { 1788) }\end{array}$ & Shikra & $C, R$ & $\checkmark$ \\
\hline 17 & $\begin{array}{l}\text { Ixobrychus sinensis } \\
\text { (Gmelin, 1789) }\end{array}$ & Yellow Bittern & U, R & & 42 & $\begin{array}{l}\text { Accipiter nisus (Linnaeus, } \\
\text { 1758) }\end{array}$ & $\begin{array}{l}\text { Eurasian } \\
\text { Sparrowhawk }\end{array}$ & $\mathrm{O}, \mathrm{M}, \mathrm{a}$ & \\
\hline 18 & $\begin{array}{l}\text { Ixobrychus cinnamomeus } \\
\text { (Gmelin, 1789) }\end{array}$ & Chestnut Bittern & U, R & $\checkmark$ & 43 & $\begin{array}{l}\text { Aquila nipalensis } \\
\text { Hodgson, } 1833\end{array}$ & Steppe Eagle & $\mathrm{U}, \mathrm{M}$ & \\
\hline 19 & $\begin{array}{l}\text { Dupetor flavicollis } \\
\text { (Latham, 1790) }\end{array}$ & Black Bittern & U, R & $\sqrt{ }$ & 44 & $\begin{array}{l}\text { Aquila clanga Pallas, } \\
1811^{\#}\end{array}$ & $\begin{array}{l}\text { Greater Spotted } \\
\text { Eagle }\end{array}$ & $\mathrm{U}, \mathrm{M}$ & \\
\hline & Ciconidae & & & & 45 & $\begin{array}{l}\text { Hieraaetus pennatus } \\
\text { (Gmelin, 1788) }{ }^{\#}\end{array}$ & Booted Eagle & $\mathrm{U}, \mathrm{M}$ & \\
\hline 20 & $\begin{array}{l}\text { Mycteria leucocephala } \\
\text { (Pennant, 1769) }\end{array}$ & Painted Stork & $\mathrm{O}, \mathrm{M}$ & & & Pandionidae & & & \\
\hline 21 & $\begin{array}{l}\text { Anastomus oscitans } \\
\text { (Boddaert, 1783) }\end{array}$ & $\begin{array}{l}\text { Asian Openbill- } \\
\text { Stork }\end{array}$ & U, M & & 46 & $\begin{array}{l}\text { Pandion haliaetus } \\
\text { (Linnaeus, 1758) }\end{array}$ & Osprey & $\mathrm{C}, \mathrm{M}$ & \\
\hline 22 & Ciconia episcopus & White-necked & U, LM & & & Falconidae & & & \\
\hline & $(\mathrm{Bc}$ & & & & 47 & Falco tinnunculus & Common Kestrel & $\mathrm{O}, \mathrm{SH}$ & \\
\hline & Threskiornidae & & & & & Linnaeus, 1758 " & & & \\
\hline 23 & \begin{tabular}{|l} 
Plegadis falcinellus \\
(Linnaeus, 1766)
\end{tabular} & Glossy lbis & $\mathrm{C}, \mathrm{M}$ & & 48 & $\begin{array}{l}\text { Falco peregrinus Tunstall, } \\
1771^{\#}\end{array}$ & Peregrine Falcon & $\mathrm{U}, \mathrm{M}$ & \\
\hline 24 & $\begin{array}{l}\text { Threskiornis melano- } \\
\text { cephalus (Latham, 1790) }\end{array}$ & $\begin{array}{l}\text { Oriental White } \\
\text { Ibis }\end{array}$ & $C, R$ & $\sqrt{ }$ & & & & & \\
\hline
\end{tabular}




\begin{tabular}{|c|c|c|c|c|}
\hline & Scientific name & Common name & Status & $\begin{array}{l}\text { Bree- } \\
\text { ding }\end{array}$ \\
\hline & Rallidae & & & \\
\hline 49 & $\begin{array}{l}\text { Amaurornis phoenicurus } \\
\text { (Pennant, 1769) }\end{array}$ & $\begin{array}{l}\text { White-breasted } \\
\text { Waterhen }\end{array}$ & $\mathrm{C}, \mathrm{R}$ & $\sqrt{ }$ \\
\hline 50 & $\begin{array}{l}\text { Rallina eurizonoides } \\
\text { (Lafresnaye, 1845) }\end{array}$ & $\begin{array}{l}\text { Slaty-legged } \\
\text { Crake }\end{array}$ & $\mathrm{U}, \mathrm{M}$ & \\
\hline 51 & $\begin{array}{l}\text { Galliarallus striatus } \\
\text { Linnaeus, } 1766^{\#}\end{array}$ & $\begin{array}{l}\text { Blue-breasted } \\
\text { Rail } \#\end{array}$ & $\mathrm{O}, \mathrm{M}$ & \\
\hline 52 & $\begin{array}{l}\text { Porzana pusilla (Pallas, } \\
\text { 1776)\# }\end{array}$ & Baillon's Crake \# & $\mathrm{O}, \mathrm{M}$ & \\
\hline 53 & $\begin{array}{l}\text { Porzana fusca (Linnaeus, } \\
\text { 1766) }\end{array}$ & $\begin{array}{l}\text { Ruddy-breasted } \\
\text { Crake }\end{array}$ & $\mathrm{U}, \mathrm{M}$ & \\
\hline 54 & $\begin{array}{l}\text { Gallicrex cinerea (Gmelin, } \\
\text { 1789) }\end{array}$ & Watercock & $\mathrm{C}, \mathrm{R}$ & \\
\hline 55 & $\begin{array}{l}\text { Porphyrio porphyrio } \\
\text { (Linnaeus, } 1758 \text { ) }\end{array}$ & Purple Moorhen & $\mathrm{C}, \mathrm{R}$ & \\
\hline 56 & $\begin{array}{l}\text { Gallinula chloropus } \\
\text { (Linnaeus, 1758) }\end{array}$ & $\begin{array}{l}\text { Common } \\
\text { Moorhen }\end{array}$ & $\mathrm{U}, \mathrm{M}$ & \\
\hline \multirow[t]{2}{*}{57} & $\begin{array}{l}\text { Fulica atra Linnaeus, } \\
1758\end{array}$ & Common Coot & $\mathrm{U}, \mathrm{M}$ ? & \\
\hline & Jacanidae & & & \\
\hline 58 & $\begin{array}{l}\text { Hydrophasianus chirurgus } \\
\text { (Scopoli, 1786) }\end{array}$ & $\begin{array}{l}\text { Pheasant-tailed } \\
\text { Jacana }\end{array}$ & $C, R$ & \\
\hline \multirow[t]{2}{*}{59} & $\begin{array}{l}\text { Metopidius indicus } \\
\text { (Latham, 1790) }\end{array}$ & $\begin{array}{l}\text { Bronze-winged } \\
\text { Jacana }\end{array}$ & $\mathrm{C}, \mathrm{R}$ & \\
\hline & Rostratulidae & & & \\
\hline \multirow[t]{2}{*}{60} & $\begin{array}{l}\text { Rostratula benghalensis } \\
\text { (Linnaeus, 1758) }\end{array}$ & $\begin{array}{l}\text { Greater Painted- } \\
\text { Snipe }\end{array}$ & $\mathrm{U}, \mathrm{R}$ & \\
\hline & Charadridae & & & \\
\hline 61 & $\begin{array}{l}\text { Pluvialis fulva (Gmelin, } \\
\text { 1789) }\end{array}$ & $\begin{array}{l}\text { Pacific Golden- } \\
\text { Plover }\end{array}$ & $\mathrm{C}, \mathrm{M}$ & \\
\hline 62 & $\begin{array}{l}\text { Pluvialis squatarola } \\
\text { (Linnaeus, 1758) }\end{array}$ & Grey Plover & $U, M$ & \\
\hline 63 & $\begin{array}{l}\text { Charadrius dubius } \\
\text { Scopoli, } 1786\end{array}$ & $\begin{array}{l}\text { Little Ringed } \\
\text { Plover }\end{array}$ & $U, M$ & \\
\hline 64 & $\begin{array}{l}\text { Charadrius alexandrinus } \\
\text { Linnaeus, } 1758\end{array}$ & Kentish Plover & $U, M$ & \\
\hline 65 & $\begin{array}{l}\text { Charadrius mongolus } \\
\text { Pallas, } 1776\end{array}$ & $\begin{array}{l}\text { Lesser Sand } \\
\text { Plover }\end{array}$ & C, M & \\
\hline 66 & $\begin{array}{l}\text { Charadrius leschemaultii } \\
\text { Lesson, } 1826\end{array}$ & $\begin{array}{l}\text { Greater Sand } \\
\text { Plover }\end{array}$ & $\mathrm{O}, \mathrm{M}$ & \\
\hline 67 & $\begin{array}{l}\text { Vanellus malabaricus } \\
\text { (Boddaert, 1783) }\end{array}$ & $\begin{array}{l}\text { Yellow-wattled } \\
\text { Lapwing }\end{array}$ & O, LM & \\
\hline \multirow[t]{2}{*}{68} & $\begin{array}{l}\text { Vanellus indicus } \\
\text { (Boddaert, 1783) }\end{array}$ & $\begin{array}{l}\text { Red-wattled } \\
\text { Lapwing }\end{array}$ & $\mathrm{C}, \mathrm{R}$ & \\
\hline & Scolopacidae & & & \\
\hline 69 & $\begin{array}{l}\text { Gallinago gallinago } \\
\text { (Linnaeus, 1758) }\end{array}$ & Common Snipe & $C, M$ & \\
\hline 70 & $\begin{array}{l}\text { Limosa limosa (Linnaeus, } \\
\text { 1758) }\end{array}$ & $\begin{array}{l}\text { Black-tailed } \\
\text { Godwit }\end{array}$ & $C, M$ & \\
\hline 71 & $\begin{array}{l}\text { Numenius phaeopus } \\
\text { (Linnaeus, 1758) }\end{array}$ & Whimbrel & $U, M$ & \\
\hline 72 & $\begin{array}{l}\text { Numenius arquata } \\
\text { (Linnaeus, 1758) }\end{array}$ & Eurasian Curlew & $U, M$ & \\
\hline 73 & $\begin{array}{l}\text { Tringa totanus (Linnaeus, } \\
\text { 1758) }\end{array}$ & $\begin{array}{l}\text { Common } \\
\text { Redshank }\end{array}$ & C, M & \\
\hline 74 & $\begin{array}{l}\text { Tringa erythropus (Pallas, } \\
\text { 1764) }\end{array}$ & $\begin{array}{l}\text { Spotted } \\
\text { Redshank }\end{array}$ & $\mathrm{O}, \mathrm{M}$ & \\
\hline
\end{tabular}

\begin{tabular}{|c|c|c|c|c|}
\hline & Scientific name & Common name & Status & $\begin{array}{l}\text { Bree- } \\
\text { ding }\end{array}$ \\
\hline 75 & $\begin{array}{l}\text { Tringa stagnatilis } \\
\text { (Bechstein, 1803) }\end{array}$ & Marsh Sandpiper & $U, M$ & \\
\hline 76 & $\begin{array}{l}\text { Tringa nebularia (Gunner, } \\
\text { 1767) }\end{array}$ & $\begin{array}{l}\text { Common } \\
\text { Greenshank }\end{array}$ & $\mathrm{C}, \mathrm{M}$ & \\
\hline 77 & $\begin{array}{l}\text { Tringa ochropus } \\
\text { Linnaeus, } 1758\end{array}$ & Green Sandpiper & $\mathrm{C}, \mathrm{M}$ & \\
\hline 78 & $\begin{array}{l}\text { Tringa glareola Linnaeus, } \\
1758\end{array}$ & Wood Sandpiper & $\mathrm{C}, \mathrm{M}$ & \\
\hline 79 & $\begin{array}{l}\text { Actitis hypoleucos } \\
\text { Linnaeus, } 1758\end{array}$ & $\begin{array}{l}\text { Common } \\
\text { Sandpiper }\end{array}$ & $\mathrm{C}, \mathrm{M}$ & \\
\hline 80 & $\begin{array}{l}\text { Calidris temminckii } \\
\text { (Leisler, 1812) }\end{array}$ & Temminck's Stint & $\mathrm{C}, \mathrm{M}$ & \\
\hline 81 & $\begin{array}{l}\text { Calidris minuta (Leisler, } \\
\text { 1812) }\end{array}$ & Little Stint & $U, M$ & \\
\hline 82 & $\begin{array}{l}\text { Calidris ferruguinea } \\
\text { (Pontoppidan, 1813) }\end{array}$ & $\begin{array}{l}\text { Curlew } \\
\text { Sandpiper }\end{array}$ & $\mathrm{O}, \mathrm{M}$ & \\
\hline 83 & $\begin{array}{l}\text { Calidris subminuta } \\
\text { (Midendorff, 1853) }\end{array}$ & Long-toed Stint \# & $\mathrm{O}, \mathrm{M}, \mathrm{a}$ & \\
\hline \multirow[t]{2}{*}{84} & $\begin{array}{l}\text { Phuilomachus pugnax } \\
\text { (Linnaeus, 1758) }\end{array}$ & Ruff & $\mathrm{O}, \mathrm{M}$ & \\
\hline & Recurvirostridae & & & \\
\hline \multirow[t]{2}{*}{85} & $\begin{array}{l}\text { Himantopus himantopus } \\
\text { (Linnaeus, 1758) }\end{array}$ & $\begin{array}{l}\text { Black-winged } \\
\text { Stilt }\end{array}$ & $U, R$ & $\sqrt{ }$ \\
\hline & Glareolidae & & & \\
\hline \multirow[t]{2}{*}{86} & $\begin{array}{l}\text { Glareola lactea } \\
\text { Temminck, } 1820\end{array}$ & Small Pratincole & U, LM & \\
\hline & Laridae & & & \\
\hline 87 & $\begin{array}{l}\text { Larus ichthyaetus Pallas, } \\
1773^{\#}\end{array}$ & Pallas's Gull \# & $\mathrm{O}, \mathrm{M}$ & \\
\hline 88 & $\begin{array}{l}\text { Larus brunnicephalus } \\
\text { Jerdon, } 1840\end{array}$ & $\begin{array}{l}\text { Brown-headed } \\
\text { Gull }\end{array}$ & $U, M$ & \\
\hline 89 & $\begin{array}{l}\text { Larus ridibundus } \\
\text { Linnaeus, } 1766\end{array}$ & $\begin{array}{l}\text { Black-headed } \\
\text { Gull }\end{array}$ & $C, M$ & \\
\hline 90 & $\begin{array}{l}\text { Gelochelidon nilotica } \\
\text { (Gmelin, 1789) }\end{array}$ & Gull-billed Tern & $\mathrm{C}, \mathrm{M}$ & \\
\hline 91 & $\begin{array}{l}\text { Sterna caspia Pallas, } \\
1770\end{array}$ & Caspian Tern & $\mathrm{O}, \mathrm{M}$ & \\
\hline 92 & $\begin{array}{l}\text { Sterna bengalensis } \\
\text { Lesson, 1831 \# }\end{array}$ & $\begin{array}{l}\text { Lesser Crested } \\
\text { Tern\# }\end{array}$ & $\mathrm{O}, \mathrm{M}$ & \\
\hline 93 & $\begin{array}{l}\text { Sterna bergii Lichtenstein, } \\
182{ }^{\#}\end{array}$ & $\begin{array}{l}\text { Large Crested } \\
\text { Tern \# }\end{array}$ & $\mathrm{O}, \mathrm{M}$ & \\
\hline 94 & $\begin{array}{l}\text { Sterna albifrons Pallas, } \\
1764^{\#}\end{array}$ & $\begin{array}{l}\text { Little/Saunders's } \\
\text { Tern }\end{array}$ & $\mathrm{O}, \mathrm{M}$ & \\
\hline 95 & $\begin{array}{l}\text { Sterna aurantia J.E. Gray, } \\
1831\end{array}$ & River Tern & O, LM & \\
\hline 96 & $\begin{array}{l}\text { Sterna fuscata Linnaeus, } \\
1766^{\#}\end{array}$ & Sooty Tern \# & V & \\
\hline 97 & $\begin{array}{l}\text { Chlidonias hybridus } \\
\text { (Pallas, 1811) }\end{array}$ & Whiskered Tern & $C, M$ & \\
\hline \multirow[t]{2}{*}{98} & $\begin{array}{l}\text { Childonias leucopterus } \\
\text { (Temminck, 1815) }\end{array}$ & $\begin{array}{l}\text { White-winged } \\
\text { Black Tern }\end{array}$ & $\mathrm{O}, \mathrm{M}$ & \\
\hline & Columbidae & & & \\
\hline 99 & $\begin{array}{l}\text { Columba livia Gmelin, } \\
1789\end{array}$ & $\begin{array}{l}\text { Blue Rock } \\
\text { Pigeon }\end{array}$ & $C, R$ & $\sqrt{ }$ \\
\hline 100 & $\begin{array}{l}\text { Streptopelia chinensis } \\
\text { (Scopoli, 1786) }\end{array}$ & Spotted Dove & O, LM & \\
\hline
\end{tabular}




\begin{tabular}{|c|c|c|c|c|}
\hline & Scientific name & Common name & Status & $\begin{array}{l}\text { Bree- } \\
\text { ding }\end{array}$ \\
\hline 101 & $\begin{array}{l}\text { Chalcophaps indica } \\
\text { (Linnaeus, 1758) }\end{array}$ & Emerald Dove & U, LM & \\
\hline 102 & $\begin{array}{l}\text { Ducula badia (Raffles, } \\
\text { 1822) }\end{array}$ & $\begin{array}{l}\text { Mountain } \\
\text { Imperial-Pigeon }\end{array}$ & $\mathrm{U}, \mathrm{SH}$ & \\
\hline \multirow[t]{2}{*}{103} & $\begin{array}{l}\text { Treron pompadora } \\
\text { (Gmelin, 1789) }\end{array}$ & $\begin{array}{l}\text { Pompadour } \\
\text { Green-Pigeon }\end{array}$ & U, LM & \\
\hline & Psittacidae & & & \\
\hline 104 & $\begin{array}{l}\text { Loriculus vernalis } \\
\text { (Sparrman, 1787) }\end{array}$ & $\begin{array}{l}\text { Indian Hanging- } \\
\text { Parrot }\end{array}$ & U, LM & \\
\hline 105 & $\begin{array}{l}\text { Psittacula krameri } \\
\text { (Scopoli, 1769) }\end{array}$ & $\begin{array}{l}\text { Rose-ringed } \\
\text { Parakeet }\end{array}$ & $C, R$ & $\sqrt{ }$ \\
\hline \multirow[t]{2}{*}{106} & $\begin{array}{l}\text { Psittacula cyanocephala } \\
\text { (Linnaeus, 1766) }\end{array}$ & $\begin{array}{l}\text { Plum-headed } \\
\text { Parakeet }\end{array}$ & $C, R$ & \\
\hline & Cuculidae & & & \\
\hline 107 & $\begin{array}{l}\text { Clamator jacobinus } \\
\text { (Boddaert, 1783) }\end{array}$ & $\begin{array}{l}\text { Pied Crested } \\
\text { Cuckoo }\end{array}$ & $\mathrm{O}, \mathrm{M}, \mathrm{a}$ & \\
\hline 108 & $\begin{array}{l}\text { Clamator coromandus } \\
\text { (Linnaeus, 1766) \# }\end{array}$ & $\begin{array}{l}\text { Red-winged } \\
\text { Crested Cuckoo \# }\end{array}$ & $\mathrm{O}, \mathrm{M}, \mathrm{a}$ & \\
\hline 109 & $\begin{array}{l}\text { Hierococcyx varius (Vahl, } \\
\text { 1797) }\end{array}$ & Brainfever Bird & C, LM & \\
\hline 110 & $\begin{array}{l}\text { Cuculus micropterus } \\
\text { Gould, } 1838\end{array}$ & Indian Cuckoo & $\mathrm{O}, \mathrm{M}$ & \\
\hline 111 & $\begin{array}{l}\text { Cacomantis passerinus } \\
(\text { Vahl, 1797) }\end{array}$ & $\begin{array}{l}\text { Indian Plaintive } \\
\text { Cuckoo }{ }^{\#}\end{array}$ & $U, M$ & \\
\hline 112 & $\begin{array}{l}\text { Eudynamis scolopacea } \\
\text { (Linnaeus, 1758) }\end{array}$ & Asian Koel & $C, R$ & $\sqrt{ }$ \\
\hline \multirow[t]{2}{*}{113} & $\begin{array}{l}\text { Centropus sinensis } \\
\text { (Stephens, 1815) }\end{array}$ & Greater Coucal & $C, R$ & $\sqrt{ }$ \\
\hline & Tytonidae & & & \\
\hline \multirow[t]{2}{*}{114} & Tyto alba (Scopoli, 1769) & Barn Owl & $C, R$ & $\sqrt{ }$ \\
\hline & Strigidae & & & \\
\hline 115 & $\begin{array}{l}\text { Otus bakkamoena } \\
\text { Pennant, } 1769\end{array}$ & $\begin{array}{l}\text { Collared Scops- } \\
\text { Owl }\end{array}$ & $U, R$ & \\
\hline 116 & $\begin{array}{l}\text { Ketupa zeylonensis } \\
\left(\text { Gmelin, 1788) }{ }^{\#}\right.\end{array}$ & $\begin{array}{l}\text { Brown Fish- } \\
\text { Owl \# }\end{array}$ & $\mathrm{U}, \mathrm{R} ?$ & \\
\hline 117 & $\begin{array}{l}\text { Strix ocellata (Lesson, } \\
\text { 1839) }\end{array}$ & $\begin{array}{l}\text { Mottled Wood- } \\
\text { Owl }\end{array}$ & $\mathrm{U}, \mathrm{R} ?$ & \\
\hline 118 & $\begin{array}{l}\text { Glaucidium radiatm } \\
\text { (Tickell, 1833) }\end{array}$ & Jungle Owlet & $C, R$ & \\
\hline 119 & $\begin{array}{l}\text { Athene brama (Temminck, } \\
\text { 1821) }\end{array}$ & Spotted Owlet & $\mathrm{U}, \mathrm{R} ?$ & \\
\hline \multirow[t]{2}{*}{120} & $\begin{array}{l}\text { Ninox scutulata (Raffles, } \\
1822 \text { ) }\end{array}$ & Brown Hawk-Owl & $U, R$ & $\sqrt{ }$ \\
\hline & Caprimulgidae & & & \\
\hline \multirow[t]{2}{*}{121} & $\begin{array}{l}\text { Caprimulgus atripennis } \\
\text { Jerdon, } 1845^{\#}\end{array}$ & Jerdon's Nightjar & $\mathrm{SH}, \mathrm{a}$ & \\
\hline & Apodidae & & & \\
\hline 122 & $\begin{array}{l}\text { Apus affinis (J.E. Gray, } \\
\text { 1830) }\end{array}$ & House Swift & C, LM & \\
\hline 123 & $\begin{array}{l}\text { Apus pacificus (Latham, } \\
\text { 1801) }\end{array}$ & Pacific Swift & $\mathrm{SH}$ & \\
\hline 124 & $\begin{array}{l}\text { Tachymarptis melba } \\
\text { (Linnaeus, 1758) }\end{array}$ & Alpine Swift & $\mathrm{U}, \mathrm{LM}$ & \\
\hline 125 & $\begin{array}{l}\text { Hirundapus giganteus } \\
\text { (Temminck, 1846) }\end{array}$ & $\begin{array}{l}\text { Brown-backed } \\
\text { Needletail-Swift }\end{array}$ & $\mathrm{U}, \mathrm{SH}$ & \\
\hline
\end{tabular}

\begin{tabular}{|c|c|c|c|c|}
\hline & Scientific name & Common name & Status & $\begin{array}{l}\text { Bree- } \\
\text { ding }\end{array}$ \\
\hline \multirow[t]{2}{*}{126} & $\begin{array}{l}\text { Cypsiurus balasiensis } \\
\text { (J.E. Gray, 1829) }\end{array}$ & Asian Palm-Swift & $C, R$ & $\sqrt{ }$ \\
\hline & Alcedinidae & & & \\
\hline 127 & $\begin{array}{l}\text { Alcedo atthis (Linnaeus, } \\
1758 \text { ) }\end{array}$ & $\begin{array}{l}\text { Small Blue } \\
\text { Kingfisher }\end{array}$ & $C, R$ & $\sqrt{ }$ \\
\hline 128 & $\begin{array}{l}\text { Ceyx erithaca (Linnaeus, } \\
1758)^{\#}\end{array}$ & $\begin{array}{l}\text { Oriental Dwarf } \\
\text { Kingfisher \# }\end{array}$ & $\begin{array}{l}\mathrm{O} \\
\mathrm{SH}, \mathrm{a}\end{array}$ & \\
\hline 129 & $\begin{array}{l}\text { Halcyon capensis } \\
\text { (Linnaeus, 1766) }\end{array}$ & $\begin{array}{l}\text { Stork-billed } \\
\text { Kingfisher }\end{array}$ & $C, R$ & \\
\hline 130 & $\begin{array}{l}\text { Halcyon smyrnensis } \\
\text { (Linnaeus, 1758) }\end{array}$ & $\begin{array}{l}\text { White-breasted } \\
\text { Kingfisher }\end{array}$ & $C, R$ & $\sqrt{ }$ \\
\hline 131 & $\begin{array}{l}\text { Halcyon pileata } \\
\text { (Boddaert, 1783) }\end{array}$ & $\begin{array}{l}\text { Black-capped } \\
\text { Kingfisher }\end{array}$ & $\mathrm{U}, \mathrm{M}$ & \\
\hline \multirow[t]{2}{*}{132} & $\begin{array}{l}\text { Ceryle rudis (Linnaeus, } \\
1758 \text { ) }\end{array}$ & Pied Kingfisher & $C, R$ & $\sqrt{ }$ \\
\hline & Meropidae & & & \\
\hline 133 & $\begin{array}{l}\text { Merops philippinus } \\
\text { Linnaeus, } 1766\end{array}$ & $\begin{array}{l}\text { Blue-tailed Bee- } \\
\text { eater }\end{array}$ & $\mathrm{C}, \mathrm{M}$ & \\
\hline 134 & $\begin{array}{l}\text { Merops orientalis Latham, } \\
1801\end{array}$ & Small Bee-eater & $C, R$ & \\
\hline \multirow[t]{2}{*}{135} & $\begin{array}{l}\text { Merops leschenaulti } \\
\text { Vieillot, } 1817^{\#}\end{array}$ & $\begin{array}{l}\text { Chestnut-headed } \\
\text { Bee-eater \# }\end{array}$ & RR, LM & \\
\hline & Coracidae & & & \\
\hline 136 & $\begin{array}{l}\text { Coracias benghalensis } \\
\text { (Linnaeus, 1758) }\end{array}$ & Indian Roller & $C, R$ & $\sqrt{ }$ \\
\hline \multirow[t]{2}{*}{137} & $\begin{array}{l}\text { Coracias garrulus } \\
\text { Linnaeus, } 1758^{\#}\end{array}$ & European Roller \# & V & \\
\hline & Bucerotidae & & & \\
\hline \multirow[t]{2}{*}{138} & $\begin{array}{l}\text { Ocyceros griseus } \\
\text { (Latham, 1790) \# }\end{array}$ & $\begin{array}{l}\text { Malabar Grey } \\
\text { Hornbill \# }\end{array}$ & $\begin{array}{c}\mathrm{O} \\
\mathrm{SH}, \mathrm{a}\end{array}$ & \\
\hline & Upupidae & & & \\
\hline \multirow[t]{2}{*}{139} & $\begin{array}{l}\text { Upupa epops Linnaeus, } \\
1758\end{array}$ & Common Hoopoe & U, LM & \\
\hline & Capitonidae & & & \\
\hline 140 & $\begin{array}{l}\text { Megalaima viridis } \\
\text { (Boddaert, 1783) }\end{array}$ & $\begin{array}{l}\text { White-cheeked } \\
\text { Barbet }\end{array}$ & $C, R$ & $\sqrt{ }$ \\
\hline \multirow[t]{2}{*}{141} & $\begin{array}{l}\text { Megalaima haemacephala } \\
\text { (P.L.S. Müller, 1776) \# }\end{array}$ & $\begin{array}{l}\text { Coppersmith } \\
\text { Barbet }\end{array}$ & $C, R$ & \\
\hline & Picidae & & & \\
\hline 142 & 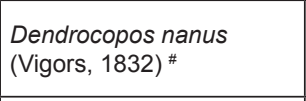 & $\begin{array}{l}\text { Brown- } \\
\text { capped Pigmy } \\
\text { Woodpecker }{ }^{\#} \\
\end{array}$ & U, LM & \\
\hline 143 & $\begin{array}{l}\text { Dendrocopos } \\
\text { mahrattensis (Latham, } \\
\text { 1801) \# }\end{array}$ & \begin{tabular}{|l|} 
Yellow-fronted \\
Pied Woodpecker \\
$\#$
\end{tabular} & $\mathrm{O}, \mathrm{R}$ & \\
\hline 144 & $\begin{array}{l}\text { Dinopium benghalense } \\
\text { (Linnaeus, 1758) }\end{array}$ & $\begin{array}{l}\text { Lesser Golden- } \\
\text { backed } \\
\text { Woodpecker } \\
\end{array}$ & $C, R$ & $\sqrt{ }$ \\
\hline \multirow[t]{2}{*}{145} & $\begin{array}{l}\text { Celeus brachyurus } \\
\text { (Vieillot, 1818) }\end{array}$ & $\begin{array}{l}\text { Rufous } \\
\text { Woodpecker \# }\end{array}$ & $\mathrm{O}, \mathrm{SH}$ & \\
\hline & Pittidae & & & \\
\hline \multirow[t]{2}{*}{146} & $\begin{array}{l}\text { Pitta brachyura (Linnaeus, } \\
\text { 1766) }\end{array}$ & Indian Pitta & UN, M & \\
\hline & Alaudidae & & & \\
\hline 147 & $\begin{array}{l}\text { Eremopterix grisea } \\
\text { (Scopoli, 1786) }\end{array}$ & $\begin{array}{l}\text { Ashy-crowned } \\
\text { Sparrow-Lark }\end{array}$ & O, LM & \\
\hline
\end{tabular}




\begin{tabular}{|c|c|c|c|c|}
\hline & Scientific name & Common name & Status & $\begin{array}{l}\text { Bree- } \\
\text { ding }\end{array}$ \\
\hline 148 & $\begin{array}{l}\text { Galerida malabarica } \\
\text { (Scopoli, 1786) }\end{array}$ & $\begin{array}{l}\text { Malabar Crested } \\
\text { Lark }\end{array}$ & O, LM & \\
\hline \multirow[t]{2}{*}{149} & $\begin{array}{l}\text { Alauda gulgula Franklin, } \\
1831\end{array}$ & Eastern Sky Lark & U, LM & \\
\hline & Hirundinidae & & & \\
\hline 150 & $\begin{array}{l}\text { Hirundo rustica Linnaeus, } \\
1758\end{array}$ & $\begin{array}{l}\text { Common } \\
\text { Swallow }\end{array}$ & $\mathrm{C}, \mathrm{M}$ & \\
\hline 151 & $\begin{array}{l}\text { Hirundo daurica Linnaeus, } \\
1771\end{array}$ & $\begin{array}{l}\text { Red-rumped } \\
\text { Swallow }\end{array}$ & C, LM & \\
\hline 152 & $\begin{array}{l}\text { Hirundo smithii Leach, } \\
1818^{\#}\end{array}$ & $\begin{array}{l}\text { Wire-tailed } \\
\text { Swallow" }\end{array}$ & U, LM & \\
\hline 153 & $\begin{array}{l}\text { Hirundo fluvicola Blyth, } \\
\text { 1855\# }\end{array}$ & $\begin{array}{l}\text { Streak-throated } \\
\text { Swallow }\end{array}$ & $\begin{array}{l}\mathrm{U}, \mathrm{LM} \\
\mathrm{a}\end{array}$ & \\
\hline 154 & $\begin{array}{l}\text { Hirundo tahitica Gmelin, } \\
1789\end{array}$ & House Swallow & $\begin{array}{c}\mathrm{O}, \\
\mathrm{SH}, \mathrm{a}\end{array}$ & \\
\hline \multirow[t]{2}{*}{155} & $\begin{array}{l}\text { Riparia diluta (Sharpe \& } \\
\text { Wyatt, 1893) }\end{array}$ & Pale Martin & $\mathrm{V}, \mathrm{a}$ & \\
\hline & Motacillidae & & & \\
\hline 156 & $\begin{array}{l}\text { Dendronanthus indica } \\
\text { (Gmelin, 1789) \# }\end{array}$ & Forest Wagtail \# & $\mathrm{C}, \mathrm{M}$ & \\
\hline 157 & $\begin{array}{l}\text { Motacilla alba Linnaeus, } \\
1758\end{array}$ & White Wagtail & $\mathrm{C}, \mathrm{M}$ & \\
\hline 158 & $\begin{array}{l}\text { Motacilla cinerea Tunstall, } \\
1771\end{array}$ & Grey Wagtail & $\mathrm{C}, \mathrm{M}$ & \\
\hline 159 & $\begin{array}{l}\text { Motacilla flava Linnaeus, } \\
1758\end{array}$ & Yellow Wagtail & $A, M$ & \\
\hline 160 & $\begin{array}{l}\text { Motacilla citriola Pallas, } \\
1776\end{array}$ & Citrine Wagtail & $\mathrm{U}, \mathrm{M}$ & \\
\hline 161 & $\begin{array}{l}\text { Motacilla } \\
\text { maderasparensis Gmelin, } \\
1789\end{array}$ & $\begin{array}{l}\text { Large Pied } \\
\text { Wagtail }\end{array}$ & $\mathrm{C}, \mathrm{R}$ & $\sqrt{ }$ \\
\hline \multirow[t]{2}{*}{162} & $\begin{array}{l}\text { Anthus rufulus Vieillot, } \\
1818\end{array}$ & Paddyfield Pipit & C, LM & \\
\hline & Campephagidae & & & \\
\hline 163 & $\begin{array}{l}\text { Coracina macei (Lesson, } \\
\text { 1830)\# }\end{array}$ & $\begin{array}{l}\text { Large Cuckoo- } \\
\text { shrike } \#\end{array}$ & U?, LM & \\
\hline 164 & $\begin{array}{l}\text { Coracina melanoptera } \\
\text { (Rüppell, 1839)\# }\end{array}$ & $\begin{array}{l}\text { Black-headed } \\
\text { Cuckoo-shrike }\end{array}$ & U?, LM & \\
\hline 165 & $\begin{array}{l}\text { Pericrocotus } \\
\text { cinnamomeus }\end{array}$ & Small Minivet & U?, LM & \\
\hline 166 & $\begin{array}{l}\text { Pericrocotus flammeus } \\
\text { (Forster, 1781) \# }\end{array}$ & Scarlet Minivet \# & U?, LM & \\
\hline \multirow[t]{2}{*}{167} & $\begin{array}{l}\text { Tephrodornis } \\
\text { pondicerianus (Gmelin, } \\
1789)^{\#}\end{array}$ & $\begin{array}{l}\text { Common } \\
\text { Woodshrike }\end{array}$ & U?, LM & \\
\hline & Pycnonotidae & & & \\
\hline 168 & $\begin{array}{l}\text { Pycnonotus jocosus } \\
\text { (Linnaeus, 1758) }\end{array}$ & $\begin{array}{l}\text { Red-whiskered } \\
\text { Bulbul }\end{array}$ & $\mathrm{U}, \mathrm{R}$ & \\
\hline \multirow[t]{2}{*}{169} & $\begin{array}{l}\text { Pycnonotus } \\
\text { cafer(Linnaeus, 1766) }\end{array}$ & $\begin{array}{l}\text { Red-vented } \\
\text { Bulbul }\end{array}$ & $C, R$ & $\sqrt{ }$ \\
\hline & Irenidae & & & \\
\hline 170 & $\begin{array}{l}\text { Chloropsis } \\
\text { cochinchinensis (Gmelin, } \\
1788)\end{array}$ & $\begin{array}{l}\text { Jerdon's } \\
\text { Chloropsis }\end{array}$ & $\mathrm{U}, \mathrm{R}$ & $\sqrt{ }$ \\
\hline 171 & $\begin{array}{l}\text { Chloropsis aurifrons } \\
\text { (Temminck, 1829) }\end{array}$ & $\begin{array}{l}\text { Gold-fronted } \\
\text { Chloropsis }\end{array}$ & O, LM & \\
\hline 172 & $\begin{array}{l}\text { Aegithina tiphia } \\
\text { (Linnaeus, 1758) }\end{array}$ & Common lora & $C, R$ & $\sqrt{ }$ \\
\hline
\end{tabular}

\begin{tabular}{|c|c|c|c|c|}
\hline & Scientific name & Common name & Status & $\begin{array}{l}\text { Bree- } \\
\text { ding }\end{array}$ \\
\hline & Lanidae & & & \\
\hline 173 & $\begin{array}{l}\text { Lanius cristatus Linnaeus, } \\
1758\end{array}$ & Brown Shrike & $U, M$ & \\
\hline \multirow[t]{2}{*}{174} & $\begin{array}{l}\text { Lanius schach Linnaeus, } \\
1758^{\#}\end{array}$ & $\begin{array}{l}\text { Rufous-backed } \\
\text { Shrike }\end{array}$ & O, LM & \\
\hline & Turdinae & & & \\
\hline 175 & Zoothera citrina cyanotus & $\begin{array}{l}\text { Orange-headed } \\
\text { Thrush }\end{array}$ & O, LM & \\
\hline 176 & $\begin{array}{l}\text { Copsychus saularis } \\
\text { (Linnaeus, 1758) }\end{array}$ & $\begin{array}{l}\text { Oriental Magpie- } \\
\text { Robin }\end{array}$ & $C, R$ & $\sqrt{ }$ \\
\hline 177 & $\begin{array}{l}\text { Saxicoloides } \\
\text { fulicata(Linnaeus, 1776) }\end{array}$ & Indian Robin & O, LM & \\
\hline 178 & $\begin{array}{l}\text { Saxicola torquata } \\
\text { (Linnaeus, 1766) }\end{array}$ & $\begin{array}{l}\text { Common } \\
\text { Stonechat \# }\end{array}$ & $\mathrm{O}, \mathrm{M}$ & \\
\hline 179 & $\begin{array}{l}\text { Saxicola caprata } \\
\text { (Linnaeus, 1766) }\end{array}$ & Pied Bushchat & O, LM & \\
\hline \multirow[t]{2}{*}{180} & $\begin{array}{l}\text { Luscinia svecica } \\
\text { (Linnaeus, 1758) })^{\#}\end{array}$ & Bluethroat \# & $\mathrm{O}, \mathrm{M}$ & \\
\hline & Timalinae & & & \\
\hline 181 & $\begin{array}{l}\text { Turdoides affinis (Jerdon, } \\
\text { 1847) }\end{array}$ & $\begin{array}{l}\text { White-headed } \\
\text { Babbler }\end{array}$ & $U, R$ & \\
\hline \multirow[t]{2}{*}{182} & $\begin{array}{l}\text { Turdoides striatus } \\
\text { (Dumont, 1823) }\end{array}$ & Jungle Babbler & $C, R$ & \\
\hline & Sylviinae & & & \\
\hline 183 & $\begin{array}{l}\text { Prinia hodgsonii Blyth, } \\
1844\end{array}$ & Franklin's Prinia & $U, R$ & \\
\hline 184 & $\begin{array}{l}\text { Cisticola juncidis } \\
\text { (Rafinesque, 1810) }\end{array}$ & $\begin{array}{l}\text { Streaked Fantail- } \\
\text { Warbler }\end{array}$ & $C, R$ & $\sqrt{ }$ \\
\hline 185 & $\begin{array}{l}\text { Prinia socialis Sykes, } \\
1832\end{array}$ & Ashy Prinia & $C, R$ & \\
\hline 186 & $\begin{array}{l}\text { Prinia inornata Sykes, } \\
1832\end{array}$ & Plain Prinia & $C, R$ & $\sqrt{ }$ \\
\hline 187 & $\begin{array}{l}\text { Acrocephalus dumetorum } \\
\text { Blyth, } 1849\end{array}$ & $\begin{array}{l}\text { Blyth's Reed- } \\
\text { Warbler }\end{array}$ & $\mathrm{C}, \mathrm{M}$ & \\
\hline 188 & $\begin{array}{l}\text { Acrocephalus stentoreus } \\
\text { (Hemprich \& Ehrenberg, } \\
\text { 1833) }\end{array}$ & $\begin{array}{l}\text { Indian Great } \\
\text { Reed-Warbler }\end{array}$ & $C, R$ & $\sqrt{ }$ \\
\hline 189 & $\begin{array}{l}\text { Orthotomus sutorius } \\
\text { (Pennant, 1769) }\end{array}$ & $\begin{array}{l}\text { Common } \\
\text { Tailorbird }\end{array}$ & $C, R$ & \\
\hline 190 & $\begin{array}{l}\text { Phyoscopus trochiloides } \\
\text { (Sundevall, 1837) }\end{array}$ & $\begin{array}{l}\text { Greenish Leaf- } \\
\text { Warbler }\end{array}$ & $C, M$ & \\
\hline \multirow[t]{2}{*}{191} & $\begin{array}{l}\text { Phyoscopus magnirostris } \\
\text { Blyth, 1843\# }\end{array}$ & $\begin{array}{l}\text { Large-billed Leaf- } \\
\text { Warbler \# }\end{array}$ & $\mathrm{O}, \mathrm{M}$ & \\
\hline & Muscicapinae & & & \\
\hline \multirow[t]{2}{*}{192} & $\begin{array}{l}\text { Muscicapa dauurica } \\
\text { Pallas, } 1811^{\#}\end{array}$ & $\begin{array}{l}\text { Asian Brown } \\
\text { Flycatcher } \#\end{array}$ & $U, M$ & \\
\hline & Monarchinae & & & \\
\hline 193 & $\begin{array}{l}\text { Terpsiphone paradisi } \\
\text { (Linnaeus, 1758) }\end{array}$ & $\begin{array}{l}\text { Asian Paradise- } \\
\text { Flycatcher }\end{array}$ & $C, M$ & \\
\hline \multirow[t]{2}{*}{194} & $\begin{array}{l}\text { Hypothymis azurea } \\
\text { (Boddaert, 1783) }\end{array}$ & $\begin{array}{l}\text { Black-naped } \\
\text { Monarch- } \\
\text { Flycatcher }\end{array}$ & $\mathrm{O}, \mathrm{SH}$ & \\
\hline & Rhipidurinae & & & \\
\hline 195 & $\begin{array}{l}\text { Rhipidura aureola Lesson, } \\
1830^{\#}\end{array}$ & $\begin{array}{l}\text { White-browed } \\
\text { Fantail- } \\
\text { Flycatcher \# }\end{array}$ & O, LM? & \\
\hline
\end{tabular}




\begin{tabular}{|c|c|c|c|c|}
\hline & Scientific name & Common name & Status & $\begin{array}{l}\text { Bree- } \\
\text { ding }\end{array}$ \\
\hline & Paridae & & & \\
\hline \multirow[t]{2}{*}{196} & $\begin{array}{l}\text { Parus major Linnaeus, } \\
1758^{\#}\end{array}$ & Great Tit \# & $C, R$ & $\sqrt{ }$ \\
\hline & Dicaeidae & & & \\
\hline \multirow[t]{2}{*}{197} & $\begin{array}{l}\text { Dicaeum erythrorh-ynchos } \\
\text { (Latham, 1790) }\end{array}$ & $\begin{array}{l}\text { Tickell's } \\
\text { Flowerpecker }\end{array}$ & $C, R$ & \\
\hline & Nectarinidae & & & \\
\hline 198 & $\begin{array}{l}\text { Nectarinia zeylonica } \\
\text { (Linnaeus, 1766) }\end{array}$ & $\begin{array}{l}\text { Purple-rumped } \\
\text { Sunbird }\end{array}$ & $C, R$ & \\
\hline 199 & $\begin{array}{l}\text { Nectarinia asiatica } \\
\text { (Latham, 1790) }\end{array}$ & Purple Sunbird & $U, R$ & \\
\hline \multirow[t]{2}{*}{200} & $\begin{array}{l}\text { Nectarinia lotenia } \\
\text { (Linnaeus, 1766) }\end{array}$ & Loten's Sunbird & $C, R$ & \\
\hline & Zosteropidae & & & \\
\hline \multirow[t]{2}{*}{201} & $\begin{array}{l}\text { Zosterops palpebrosus } \\
\text { (Temminck, 1824)\# }\end{array}$ & $\begin{array}{l}\text { Oriental White- } \\
\text { eye }^{\#}\end{array}$ & $\mathrm{O}, \mathrm{SH}$ & \\
\hline & Estrildidae & & & \\
\hline 202 & $\begin{array}{l}\text { Lonchura striata } \\
\text { (Linnaeus, 1766) }\end{array}$ & $\begin{array}{l}\text { White-rumped } \\
\text { Munia }\end{array}$ & $C, R$ & \\
\hline 203 & $\begin{array}{l}\text { Lonchura punctulata } \\
\text { (Linnaeus, 1758) }\end{array}$ & Spotted Munia & $U, R$ & \\
\hline \multirow[t]{2}{*}{204} & $\begin{array}{l}\text { Lonchura malacca } \\
\text { (Linnaeus, 1766) }\end{array}$ & $\begin{array}{l}\text { Black-headed } \\
\text { Munia }\end{array}$ & U, LM & \\
\hline & Passeridae & & & \\
\hline 205 & $\begin{array}{l}\text { Passer domesticus } \\
\text { (Linnaeus, 1758) }\end{array}$ & House Sparrow & $U, \mathrm{R}$ & $\sqrt{ }$ \\
\hline \multirow[t]{2}{*}{206} & $\begin{array}{l}\text { Petronia xanthocollis } \\
\text { (Burton, 1838) \# }\end{array}$ & $\begin{array}{l}\text { Yellow-throated } \\
\text { Sparrow }\end{array}$ & $\begin{array}{l}\mathrm{U}, \mathrm{M} \\
(\mathrm{BV})\end{array}$ & $\sqrt{ }$ \\
\hline & Ploceinae & & & \\
\hline 207 & $\begin{array}{l}\text { Ploceus philippinus } \\
\text { (Linnaeus, 1766) }\end{array}$ & Baya Weaver & $C, R$ & $\sqrt{ }$ \\
\hline \multirow[t]{2}{*}{208} & $\begin{array}{l}\text { Ploceus manyar } \\
\text { (Horsfield, 1821) }\end{array}$ & Streaked Weaver & $U, R$ & $\sqrt{ }$ \\
\hline & Sturnidae & & & \\
\hline 209 & $\begin{array}{l}\text { Sturnus malabaricus } \\
\text { malabaricus (Gmelin, } \\
1789)\end{array}$ & $\begin{array}{l}\text { Grey-headed } \\
\text { Starling }\end{array}$ & $\mathrm{C}, \mathrm{M}$ & \\
\hline 210 & Sturnus malabaricus blythi & Blyth's Myna & $\mathrm{O}, \mathrm{SH}$ & \\
\hline 211 & $\begin{array}{l}\text { Acridotheres tristis } \\
\text { (Linnaeus, 1766) }\end{array}$ & Common Myna & $C, R$ & $\sqrt{ }$ \\
\hline 212 & $\begin{array}{l}\text { Acridotheres fuscus } \\
\text { (Wagler, 1827) }\end{array}$ & Jungle Myna & $C, R$ & $\sqrt{ }$ \\
\hline 213 & $\begin{array}{l}\text { Sturnus roseus (Linnaeus, } \\
\text { 1758)\# }\end{array}$ & Rosy Starling & $U, M$ & \\
\hline \multirow[t]{2}{*}{214} & $\begin{array}{l}\text { Sturnus pagodarum } \\
\text { (Gmelin, 1789) }\end{array}$ & $\begin{array}{l}\text { Brahminy } \\
\text { Starling }\end{array}$ & $\mathrm{O}, \mathrm{M}$ & \\
\hline & Oriolidae & & & \\
\hline 215 & $\begin{array}{l}\text { Oriolus oriolus (Linnaeus, } \\
1758 \text { ) }\end{array}$ & $\begin{array}{l}\text { Eurasian Golden } \\
\text { Oriole }\end{array}$ & $C, M$ & \\
\hline 216 & $\begin{array}{l}\text { Oriolus chinensis } \\
\text { Linnaeus, } 17666^{\#}\end{array}$ & $\begin{array}{l}\text { Black-naped } \\
\text { Oriole } \#\end{array}$ & $\mathrm{O}, \mathrm{M}$ & \\
\hline 217 & $\begin{array}{l}\text { Oriolus xanthornus } \\
\text { (Linnaeus, 1758) }\end{array}$ & $\begin{array}{l}\text { Black-headed } \\
\text { Oriole }\end{array}$ & $C, R$ & $\sqrt{ }$ \\
\hline
\end{tabular}

\begin{tabular}{|c|c|c|c|c|}
\hline & Scientific name & Common name & Status & $\begin{array}{l}\text { Bree- } \\
\text { ding }\end{array}$ \\
\hline & Dicruridae & & & \\
\hline 218 & $\begin{array}{l}\text { Dicrurus macrocercus } \\
\text { Vieillot, } 1817\end{array}$ & Black Drongo & $C, R$ & $\sqrt{ }$ \\
\hline 219 & $\begin{array}{l}\text { Dicrurus leucophaeus } \\
\text { Vieillot, } 1817\end{array}$ & Ashy Drongo & $\mathrm{O}, \mathrm{M}$ & \\
\hline 220 & $\begin{array}{l}\text { Dicrurus aeneus Vieillot, } \\
1817^{\#}\end{array}$ & Bronzed Drongo \# & U, LM & \\
\hline \multirow[t]{2}{*}{221} & $\begin{array}{l}\text { Dicrurus paradiseus } \\
\text { (Linnaeus, 1766) })^{\#}\end{array}$ & $\begin{array}{l}\text { Greater Racket- } \\
\text { tailed Drongo \# }\end{array}$ & $C, R$ & \\
\hline & Artamidae & & & \\
\hline \multirow[t]{2}{*}{222} & $\begin{array}{l}\text { Artamus fuscus Vieillot, } \\
1817\end{array}$ & $\begin{array}{l}\text { Ashy } \\
\text { Woodswallow }\end{array}$ & $C, R$ & $\sqrt{ }$ \\
\hline & Corvidae & & & \\
\hline 223 & $\begin{array}{l}\text { Dendrocitta vagabunda } \\
\text { (Latham, 1790) }\end{array}$ & Indian Tree Pie & $C, R$ & $\sqrt{ }$ \\
\hline 224 & $\begin{array}{l}\text { Corvus splendens Vieillot, } \\
1817\end{array}$ & House Crow & $C, R$ & $\sqrt{ }$ \\
\hline 225 & $\begin{array}{l}\text { Corvus macrorhynchos } \\
\text { Wagler, } 1827\end{array}$ & Jungle Crow & $C, R$ & \\
\hline
\end{tabular}

R - Resident; M - Migrant; LM - Local Migrant; SH - Straggler from hill; V - Vagrant; A - abundant; C - Common; UN - Uncommon; O - Occasional; BV - Breeding visitor; a - Sightings made by others but validated by author(s); \# - Not recorded from Kole wetlands;

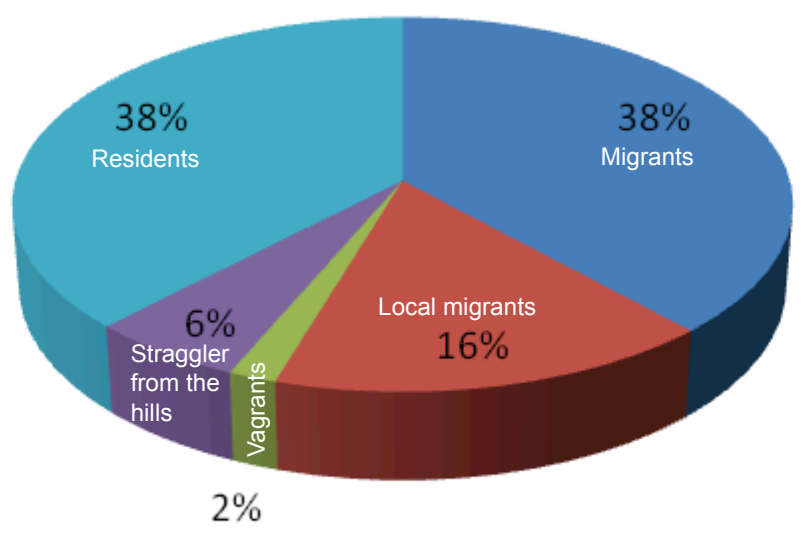

Figure 2. Status of the birds recorded from the Kuttanad wetlands

melanogaster, Greater Grey-headed Fish-Eagle Ichthyophaga ichthyaetus, Black-tailed Godwit Limosa limosa, Eurasian Curlew Numenius arquata and European Roller Coracias garrulus are listed in the Near Threatened category (IUCN 2010).

Among the 225 species of birds recorded, 55 species are found to breed in the Kuttanad wetlands. Kumarakom heronry is the biggest of all heronries reported from Kuttanad and so far 12 species of wetland birds were found to breed in this heronry. The Near Threatened Oriental Darter and Oriental 


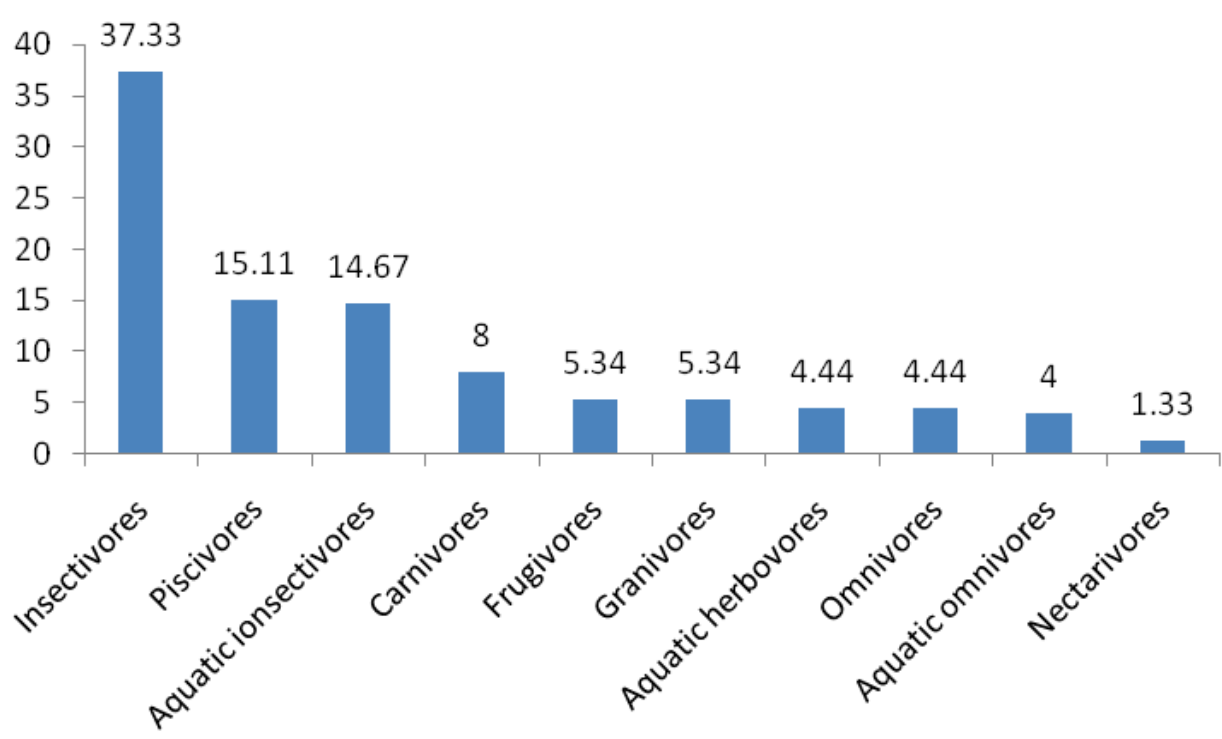

Figure 3. Percentage distribution of feeding guilds of birds in Kuttanad wetland
White Ibis were found to breed during monsoon. Among the colonial nesting waterbirds Oriental White Ibis, Indian Shag Phalacrocorax fuscicollis, Large Egret Casmerodius albus, Median Egret Mesophoyx intermedia were found to breed only in the Kumarakom heronry, and Little Cormorants Phalacrocorax niger and Indian Pond Heron Ardeola grayii were found to nest in more than two areas. Kuttanad wetland support relatively large flocks of Egret spp., Oriental White Ibis, Little Cormorants, Indian Shag, Darter, Glossy Ibis Plegadis falcinellus.

The birds that are of interest owing to their rarity as far as Kerala is concerned, and which were sighted during the period of the study period from Kuttanad region are given below.

\section{Sightings of special interest}

Oriental Darter Anhinga melanogaster - Zacharias \& Gaston (2003) reported that the Oriental Darter population had declined in Kerala during last three decades. But it is one among the common species of wetland bird of this wetland. Narayanan \& Vijayan (2007) recorded about $8 \%$ of the South Asian population of Oriental Darter during the breeding season of 2004.

Painted Stork Mycteria leucocephala - A flock of six birds were sighted at Parippu in the Kayal Kuttanad region on 05 January 2000. There were only a handful of sightings of this species from Kerala during the period of study.

Eurasian Spoonbill Platelia leucorodia - four sightings from Kayal and Lower Kuttanad. Solitary bird in a paddy field close to the MoncompuChampakkulam road near Moncompu on 29November 1996; one at Judgy Aarayiram paddy fields on 13 April 2003 (K.M. Sajith pers. comm. 2003); one individual on flight at Ramankary on 21 September 2003 and on the same day Dipu Sasi (pers. obs.) saw a flock of seven individuals on flight at Kumarakom.

Spotted Redshank Tringa erythropus - A loose flock of 10 birds were located (04 November 2001) on the mud flats of Erupathinaalayiram paddy field at Kayal Kuttanad. This formed the second sight record of this species from Kerala State.

Long-toed Stint Calidris subminuta - Sighted and photographed by Sathyan Meppayur, Tim Inskipp and Carol Inskipp from Pathinaalayiram paddy fields on 04 December 2006 (Sathyan Meppayur pers. comm. 2008). This could be the first report of this species from Kerala.

Ferruginous Pochard Aythya nyroca - One male individual of this species has been sighted by the second author on December 1976 along with a Brahminy Shelduck Tadorna ferruginea between Pathiramanal Island and Thannermukkom Bund. This is a very rare duck species and has very few records so far from Kerala State.

Sooty Tern Sterna fuscata - A dead specimen with a ring on the tarsi, having the ring number DB26299 was found on 03 July 1998 at Puthenchantha, near Vaakathanam, Kottayam (9030’N \& 76032'E). The same was ringed at Bird Island, Seychelles $\left(3^{0} 41^{\prime} \mathrm{N}\right.$ 
$\left.\& 55^{0} 13^{\prime} \mathrm{E}\right)$ by British Museum on 08 September 1997 and this bird has moved $2800 \mathrm{~km}$ in 328 days (Dave Anning in litt. 17 August 2001).

White-winged Black Tern Childonias leucopterus - Total three sightings of this species from Kuttanad. Eight individuals of these birds were seen on Vembanad Lake on 11 April 2003, a loose flock of 12 individuals on 13 April 2003 and two individuals at Chama paddy field near Neelamperoor on 02 October 2003.

Steppe Eagle Aquila nipalensis - Recorded from Vembanad Lake on $19^{\text {th }}$ January 2003 and Kavanattinkara on 21 January 2007. Mainly a winter visitor to the northern areas of the country.

Greater Spotted Eagle Aquila clanga - Four individuals were recorded on three occasions. Two individuals were recorded from Pallathuruthy in the Lower Kuttanad region on 19 January 2003; one individual at Kaippuzhamuttu on 18 January 2004 and the last one was from Thollayiram Kayal (16 January 2005). Mainly a winter visitor to the northern areas of the country.

Greater Grey-headed Fish-Eagle Icthyophaga icthyaetus - This species has been recorded from Pathiramanal Island in the Vembanad Lake by Sethumadhavan C.P. and Saju Vasan on 20 January 2002. This is the only record of this species from the Kuttanad part of Vembanad Lake, even though Saju Vasan has another sighting of the same from the Chithrappuzha, Ernakulam District.

Mountain Imperial Pigeon Ducula badia - These are mainly a birds of the forest biotope and it seldom seen on the wetlands. An uncommon bird - first record of this was in 05 August 2003. After the first record, regular sightings during monsoon seasons from the Kayal Kuttanad and North Kuttanad.

Malabar Grey Hornbill Ocyceros griseus - An endemic species of Western Ghats. One individual recorded on 15 January 2005 by P. Manoj (pers. comm. 2005) at Kumarakom heronry. A small relict population of this species is still surviving at Ponthanpuzha forest in the midlands of Kottayam District. Individual sighted from Kumarakom would be from the Ponthanpuzha population.

European Roller Coracias garrulus - One individual was seen at Erupathinaalayiram paddy fields of Kayal Kuttanad, 29September 2002. This was the first report of this species from Kerala State.

Oriental Dwarf Kingfisher Ceyx erithaca - One individual of this forest species were recorded from Kumarakom heronry on 16February 2005 by P. Manoj (pers. comm. 2005).

Rufous Woodpecker Celeus brachyurus - The species was recorded only once from Chennithala in the Upper Kuttanad region on 23 February 1997.

Large-billed Leaf-Warbler Phylloscopus magnirostris - Once heard on 10 January 2004 from the tree-dominated area of Ayamanam. This is essentially a bird of the evergreen forests of the hills.

Pacific Swift Apus pacificus - A huge flock of around 2000 individuals from Kumarakom and adjacent areas (north Kuttanad and Vaikomkari divisions) by David V. Raju (pers. comm. 2006) and various visiting birders to this area on 24-26 December 2006 and a flock of more than 500 individuals on 29 December 2006.

Pale Martin Riparia diluta - Sighted and photographed by Sathyan Meppayur, Tim Inskipp and Carol Inskipp from Pathinaalayiram paddy fields on 04 December 2006 (Sathyan Meppayur pers. comm. 2008). This is the first report of this species from Kerala.

\section{Major threats to the avifauna of this region}

Thanneermukkom salt-water barrage $(1250 \mathrm{~m}$ long) commissioned in 1975 was constructed across the narrow portion of the Vembanad Lake to prevent the saltwater intrusion during summer from sea and to spill out floodwater during monsoon. The Thanneermukkom barrage has greatly influenced the ecology of the region. When this regulator is closed, there is virtually no flow of water beyond it on the southern side making the entire Kuttanad a static pool. At present the barrage is open from 22 December to 22 March. Water with heavy loads of pesticides and fertilizers from the paddy fields were drained into this stagnant water body. Persistence of these kinds of situations triggered several ecological backlashes like proliferation of weed growth, deterioration of the water quality, increased morbidity among the local fish population and destruction of subsistence fishery on which the local fishermen depended (Abhilash et al. unpublished). Barrage also impaired the migration of marine and estuarine fauna. Horizontal and vertical shrinkage of Vembanad Lake, vanishing mangroves, eutrophication, increased interventions in the area by tourism, Thottappally spillway, sewage and industrial pollution etc. are the major problems of Kuttanad 
wetlands (Abhilash et al. unpublished). The loss of habitat through direct and indirect anthropogenic activities causes immense threat to the birds especially the migratory birds of Kuttanad. The most important threats to the wetland birds are the following.

Landscape alteration: The key threat factor is the landscape alteration in Kuttanad. Encroachment of the wetlands for the construction of new buildings and settlements are very common in Kuttanad, even though it is legally banned. Before and after the year 1947, huge area of the Vembanad Lake has been converted to paddy fields to enhance the rising demand for food. This adversely affected the migratory birds such as ducks and teals by reducing available roosting place, foraging areas and food in the lake. Thus the formation of mudflats in the reclaimed areas provided ideal foraging areas for the wintering waders.

Hunting: Hunting pressure is intense in some areas of Kuttanad, Sashikumar \& Palot (2002) have earlier reported heavy poaching of the birds from this area. The main hunting methods involve a combination of shooting, with hooks and line using fishes as bait, picking of nestlings from nests, especially from heronries. The main species caught by shooting is winter migrants like ducks, godwits and the species belonging to the ardeidae family. Black-crowned Night-Herons and egrets are the species heavily trapped by hook and line method (S. Dipu pers. comm. 2004). Heavy poaching of nestlings of Purple Heron from the nests situated in the thickets of Phargmites karka is prevailing in the "R Block" area.

Overgrowth of exotic vegetation: Infestation of the exotic waterweeds like Eichhornia crassipes, Slavinia molesta are causing serious harms to the water birds (Sashikumar \& Palot 2002), but at the same time this provides foraging areas for the species like Jacanas and Moorhens. Exotic vegetation also poses immense threats to the native flora and fauna of this region. Vembanad Lake is covered by Eichhornia crassipes which is drastically affecting the life of fishermen and local people, who are dependent on this lake for their basic needs.

Pesticides: Intensive use of chemical fertilizers and pesticide as a part of the agriculture activities have played havoc on the traditional farming system and life style of Kuttanad, affecting birds, other wildlife as well as human beings (Sashikumar \& Palot 2002). Organochlorine and organophosphate pesticides are widely used in paddy cultivation all over the state. Studies conducted in the Kuttanad ecosystem show that these chemicals are present well above the permissible limits. Seedikkoya \& Shukkur (2004) reported the presence of organochlorines such as DDT, DDE, Dieldrin, Aldrin and heavy metals such as zinc $(\mathrm{Zn})$ and copper $(\mathrm{Cu})$ in the Indian Pond Heron, Little Egret Egretta garzetta and Cattle Egret Bubulcus ibis from northern Kerala. But such studies on birds are not yet conducted in Kuttanad.

Felling of nesting and roosting trees: Cutting down of tall trees used by colonial nesting waterbirds is rampant in Kuttanad, and small heronries in Kerala face similar threats from local people. Loss of local flora is huge in Kuttanad wetland. The reduction in size or the total felling of sacred groves has also created trouble in the form habitat loss for land birds and wetland birds; which uses tiny patches for feeding, roosting and nesting. The extent of mangrove trees in the Kumarakom heronry, Pathiramanal has drastically reduced. After the taking over of Kumarakom heronry by KTDC, 13 hectares of the land was given to a venture company by KTDC. They cleared the mangrove belt along the lake and converted them into a lawn and constructed a tourist jetty. With this, a species of mangrove Kandelia candel was totally wiped out from the Kumarakom heronry (Ramachandran \& Mohanan 1990; Sreekumar 2001).

Tourism: Ali (1984) reported that large flocks of wintering ducks roost in the calm waters of Vembanad Lake. At present, amplified promotion of tourism by using boats and speedboats force migratory ducks to desert roosting place in the lake. Most of the tourist resorts in and around the Vembanad Lake and Kuttanad do not have any proper solid waste and waste-water treatment facilities, therefore all waste materials are dumped into the lake during night hours. This activity adversely affects self-sustaining capacity of the Lake. House boats discharge effluents and wastes directly into the lake and large amount of oil are spilled into the system. In the name of tourism the authorities, which owns the Kumarakom heronry clear pure stands of Phragmites karka, where Black-crowned Night-Heron Nycticorax nycticorax, Median Egret Mesophoyx intermedia, and Large Egret Casemerodius albus nests. This activity has drastically affected nesting habitats of the breeding birds.

Kuttanad wetlands show high diversity in terms of 
number of species especially the wetland birds; this is higher than the total terrestrial species and total wetland species recorded from the Kole wetland of Kerala (Sivaperuman \& Jayson 2000). Thirty-eight species from the Kuttanad wetlands are not recorded from the Kole wetlands (Table 1). According to Kumar et al. (2005), 128 wetland birds were included in the revised Indo-Russian agreement on the migratory birds list; of this 50 species were recorded from different parts of Kuttanad wetlands during the present study. In Upper Kuttanad region and the eastern boundary of Kuttanad, tree diversity is high compared to the main wetland. Hence most of the land birds were recorded from this area, some were restricted this area alone.

Indian Shag, Large Egret, Median Egret were found to breed only in the Kumarakom heronry; loss of nesting habitat and the disturbance caused by increased anthropogenic activities throughout the Kuttanad wetlands may be reason for this situation. Little Cormorants and Indian Pond-Heron were found to nest in more than two nesting areas. Little Cormorants and Indian Pond Heron may have the capacity to withstand the disturbance caused by human beings; this could be the possible explanation for the use of more than two nesting sites compared to the other colonial nesting water birds, but most of the other colonial nesting water bird species are congregating at Kumarakom Heronry for breeding. During the 2004 breeding season 157 nests of Darter were reported and 276 birds were counted in a single count (Narayanan 2004). According to Rahmani et al. (2002) world population of Darter was estimated as 10,000. Kumarakom heronry holds about $8 \%$ of the South Asian biogeographical population of Darter (Narayanan \& Vijayan 2007), which qualifies Criterion 6 of the Convention on Wetlands of International Importance (Ramsar Convention). Kumarakom heronry has the biggest known breeding birds of Oriental White Ibis from Kerala (Narayanan 2004). Although the Ramsar site designated in 2002 includes both Vembanad Lake and Kole wetlands, there is no definite connection between Vembanad and the Kole wetlands, and both have the potential to become independent Ramsar sites (Narayanan \& Vijayan 2007). Hence, we propose that Vembanad Lake should be declared as a Ramsar site in its own right.

During the winter months massive flocks of Glossy Ibis, which contains 500 or more birds visit the mud fields in the paddy fields and the $1 \%$ threshold of Glossy Ibis is 250 (Kumar et al. 2005). Other than Darter, species such as Oriental White Ibis, Indian Shag, Black-crowned Night Heron, and Little Cormorant in the Kumarakom heronry during the breeding season is well above the limit of $1 \%$ biogeographic population (Narayanan 2004).

\section{Conservation action plan}

The following action plan is proposed for the conservation of birds and wetlands of Kuttanad.

a. Active patrolling should be carried out by the forest department, at least five groups with four forest guards are recommended for patrolling at different parts of the Vembanad area to stop poaching.

b. Nature awareness programmes regarding birds, mangrove forests and importance of wetland ecosystem for daily sustenance of life to be given to the local people for the conservation of this eco-system. A documentary film could be produced to with the prediction of future major changes in the Kuttanad titled "Kuttanad - after twenty years". This will give a grim picture with a clear message to the people.

c. Elevate the status of Kumarakom heronry to a full-fledged sanctuary as per Wildlife (Protection) Act for the protection of 11 species of breeding birds and its unique nesting habitat.

d. "Pathiramanal", an island in the Vembanad Lake, should be protected giving a special status as "community reserve" for preserving the typical flora and fauna of the Kuttanad region.

e. Regulation of inflow and outflow from the Thanneermukkom saltwater barrage should be done properly according to the management strategies proposed in the earlier published works.

f. Reclamation of wetlands for industrial, settlement, plantation and cultivation purposes should be restricted and Government departments should be persuaded to have strict environmental impact assessment (EIA) before the implementation of any new projects in the area.

g. Restoration of mangroves in the area should be executed. For the production of new plants, vegetative propagation and tissue culture methods can be opted.

h. Remaining sacred groves must be preserved under the guidance of forest department and local owners like temple authorities.

i. Solid waste and wastewater treatment facilities 
must be developed in all tourist resorts in the Vembanad region.

j. All tourist establishments must provide a fraction of money from the yearly profit for the restoration activities of the Kuttanad wetlands through government and local NGO's.

k. Mobile checking facilities should be initiated to seize polluting boats and its license should be withheld.

1. A detailed study on the movements of the birds of this area should be conducted during various seasons to determine the spatial and temporal pattern of bird migration and ecological reasons should be identified to determine the drastic reduction in the population of many bird groups.

\section{CONCLUSIONS}

This study increased the information and knowledge available on the avifauna of Kuttanad wetlands. Kuttanad wetland is rich in wetland bird species. Degradation of this unique wetland ecosystem, hunting and habitat alteration is still prevailing in this part of the Vembanad-Kole Ramsar this threatens the birdlife directly as well as indirectly. As Kole wetlands, Kuttanad wetland is also serving as halting area for the trans-continental migrants; urgent measures should be taken to protect this wetland ecosystem for the conservation of birds especially migratory and breeding colonial nesting birds. This region holds more than the estimated number of South Asian biogeographical population of six species of waterbirds. At present the low lands of Kerala are under high threat of landscape modification due to population growth, tourism and other infrastructure developmental activities. Regular monitoring of wetland should be taken up. In-depth studies on the avifauna, especially endangered birds, should be undertaken. Hence urgent conservation measures have to be implemented and a protected area has to be evolved for preserving the remaining tract of mangroves and faunal heritage of this unique region. Local people should be made aware of the importance of wetlands, waterfowl (Sashikumar \& Palot 2002) and other common birds. Without the involvement of common people of this region conservation of the wetlands will not be successful.

\section{REFERENCES}

Abhilash, P.C., S.P. Narayanan \& B. Ajayakumar (Unpublished). The Kuttanad wetland ecosystem: an endangered wetland system of Kerala, 24pp.

Ali, S. \& S.D. Ripley (1987). Handbook of the Birds of India and Pakistan I-X Vol. Compact Edition. Oxford University Press, New Delhi, 737pp.

Ali, S. (1984). Birds of Kerala. 2nd Edition. Oxford University Press, New Delhi, 444pp.

Chandy, M. (2003). Study on the avifauna of rice fields in and around Vembanad Lake. PhD Dissertation, Mahatma Gandhi University, Kottayam, Kerala.

Grewal, B., B. Harvey \& O. Pfister (2002). A Photographic Guide to Birds of India and the Indian Subcontinent. Periplus Edition (HK) Ltd. Singapore, 513pp.

Grimmet, R., C. Inskipp \& T. Inskipp (2000). Pocket guide to the birds of the Indian Subcontinent. Oxford University Press, New Delhi, 384pp.

Indo-Dutch Mission (1989). Kuttanad water balance study plant report. Government of Kerala, Trivandrum, Kerala, 70 pp.

Islam, Z.M \& A.R. Rahmani (eds.) (2004). Important Bird Areas in India: Priority for Conservation. Bombay Natural History Society, Mumbai, xviii+1133pp.

IUCN (2010). IUCN Red List of Threatened Species. Version 2010. <www.iucnredlist.org>. Downloaded on 29 March 2010.

Kumar, A., J.P. Sati, P.C. Tak \& J.R.B. Alfred (2005). Handbook on Indian Wetland Birds and their Conservation. Zoological Survey of India, 468pp.

Maltby, E. \& R.E. Turner (1983). Wetlands of the world. Geographical Magazine 55: 12-17.

Maltby, E.R. (1986). Waterlogged Wealth: Why Waste the World's Wet Places?. Earth scan, London, UK, 132pp.

Manakadan, R. \& A. Pittie (2002). Standardized English and common names of the birds of the Indian sub-continent -2002. Newsletter for Bird Watchers 42(3): 1-36.

Mitsch, W.J. \& Gosselink (2000). Wetlands. John Wiley \& Sons Inc, United States of America, 356pp.

Nameer, P.O., R.R. Nair, K.R. Anoop, S.G. Nair, S. Lekshmi \& P. Radhakrishnan (2000). Birds of Kerala Agricultural University campus, Thrissur. Zoos' Print Journal 15(4): 243-246.

Narayanan, S.P. \& L. Vijayan (2007). Status of the colonial breeding waterbirds in Kumarakom heronry in Kerala, Southern India. Podoces 2(1): 22-29.

Narayanan, S.P. (2004). Kuttanadan pakshinamamgal (bird names of Kuttanad). Malabar Trogon 2(3): 2-3. (In Malayalam).

Narayanan, S.P., S. Dipu \& L. Vijayan (2005a). Breeding of Black-winged Stilt in Kumarakom part of Vembanad Lake, Kerala. Indian Birds 1(5): 116.

Narayanan, S.P., M.S Sajith, S. Dipu, Ajay \& B. Sreekumar (2005b). Sightings of Mountain Imperial Pigeon Ducula 
badia from the Vembanad wetlands. Malabar Trogon 3(2): 13.

Neelakantan, K.K. (1996). Keralathile pakshikal (Birds of Kerala). Kerala Sahithya Academy, Trichur, (In Malayalam), 523pp.

NEST (1993). Birds of Vembanad Lake: a survey report. Nature Education Society, Thrissur, Vembanad Nature Club, Muhamma, Alappuzha and Kerala Forest Department, Kerala, 18pp.

Rahmani, A., S. Laad, Z. U. Islam \& A. Malekar (eds.). (2002). Asian water bird census. Final report, India. Bombay Natural History Society, Bombay, 34pp.

Rakesh, P.S., S.P. Narayanan \& A.P. Thomas (2004). A study on the avifauna of Aymanam Panchayath, in Vembanad wetland, Kerala. Paper presented in National Seminar on Biodiversity Conservation. Sanadhana Dharma College, Alappuzha, Kerala, $7 \mathrm{pp}$.

Ramachandran, K.K. \& C.N. Mohanan (1990). The mangrove ecosystems in Kerala. Final report. Centre for earth science studies. Trivandrum, 38pp.

Sashikumar, C. \& M.J. Palot (2002). Wetlands and waterfowls of Kerala, India - an Overview. World conservation status of storks, ibises and spoonbills, pp.147-153. In: Rahmani, A.R \& G. Ugra (eds.). Birds of Wetlands and Grasslands. Proceedings of the Salim Ali centenary seminar Bombay Natural History Society, 360pp.

Seedikkoya, K. \& E.A.A. Shukkur (2004). Pesticide and heavy metal contamination in wetland birds, p.126. In: Marler, P. \& D. Bhatt (eds.). Abstracts Volume of international Conference on Bird and Environment. Gurukula Kangri University, Haridwar, India, 226pp

Shari, P.V. \& K.P. Chitra (2005). Kuttanadinte charithravum bhoomisastravum (History and geography of Kuttanad), pp. 9-18. In: Joseph, M.J. (ed.). Kuttanattile jala punarujjeevana prayoga sadhyathakal (Water reclaiming in Kuttanad; Possible chances). Planet Kerala, Trivandrum, 131pp. (In Malayalam).

Sivaperuman, C. \& E.A. Jayson (2000). Birds of Kole wetlands, Thrissur, Kerala. Zoos' Print Journal 15(10): 344-349.

Sreekumar, B. \& S.P. Narayanan (2004). A comparative study on the number of the wetland birds in the KTDC tourist complex Kumarakom during 2001-2004. Paper presented in National seminar on the biodiversity conservation. Sanatana Dharma college, Alappuzha, Kerala.

Sreekumar, B. (ed.) (2001). Vembanad Water Bird Count 2001. Kerala Forest and Wildlife Department, Kottayam, 30pp.

Sreekumar, B. (ed.) (2002). Vembanad Water Bird Count 2002. Kerala Forest and Wildlife Department, Kottayam, 32pp.

Sreekumar, B. (ed.) (2003). Vembanad Water Bird Count 2003. Kerala Forest and Wildlife Department, Kottayam, 80pp.

Sreekumar, B. (ed.) (2004). Vembanad Water Bird Count 2004. Kerala Forest and Wildlife Department, Kottayam, 43pp.

Sreekumar, B. (ed.) (2005). Vembanad Water Bird Count 2005. Kerala Forest and Wildlife Department, Kottayam, 48pp.

Stewart, R.E. (2001). Technical Aspects of Wetlands - Wetlands as Bird Habitat. National Water Summary on Wetland Resources. United States Geological Survey, 86pp.

Sudhikumar, A.V. \& P.A. Sebastian (2005). Diversity of spiders in Kuttanad rice agro-ecosystem, Kerala. Journal of the Bombay Natural History Society 102(1): $66-68$.

Unni, K.S. (2002). Wetlands of India. pp 1-5. In: Proceedings of the National seminar on ecology and conservation of wetlands. Limnological Association of Kerala, $102 \mathrm{pp}$.

Weller, M.W. (1999). Wetland Birds Habitat Resources and Conservation Implications. Press syndicate of the University of Cambridge, United Kingdom, 137pp.

Zacharias, V.J. and A.J. Gaston. (2003). Survey of Kerala wetlands, India. $O B C$ Bulletin 37: 15.
Author Details: S. Prasanth Narayanan is a research scholar at Mahatma Gandhi University. As part of his $\mathrm{PhD}$ programme, he is working on the ecology of the Oriental Darter of Kuttanad wetlands.

A.P. Thоmas is the Director of the Advanced Centre of Environmental Studies and Sustainable Development, an inter-university centre of Mahatma Gandhi University. He has contributed more than 50 research papers in the field of environmental sciences.

B. SREEKUMAR is a professional orthopaedic surgeon and the President of Kottayam Nature Society. Since 2001, who regularly conducts the annual waterfow census at Vembanad Lake and adjacent regions, with the help of Kerala Forest and Wildlife Department.

Author Contribution: SPN collected data and wrote major portion of the manuscript. BS helped in collecting the data and partially assisted in the preparation of the manuscript. APT assisted in writing the manuscript and provided all facilities during the preparation of the manuscript. 